\title{
Evaluation of the roles and regulatory mechanisms of PD-1 target molecules in NSCLC progression
}

\author{
Yun-Qiang Zhang ${ }^{1 \#}$, Ye Yuan ${ }^{2 \#}$, Jun Zhang ${ }^{2}$, Cheng-Yi Lin ${ }^{2}$, Jia-Long Guo ${ }^{2}$, Hua-Song Liu ${ }^{2}$, Qiang Guo ${ }^{2,3}$ \\ ${ }^{1}$ Department of Thoracic Surgery, Beilun District People's Hospital of Ningbo, Ningbo, China; ${ }^{2}$ Department of Cardiothoracic Surgery, Taihe \\ Hospital, Hubei University of Medicine, Shiyan, China; ${ }^{3}$ Department of Thoracic Surgery, Union Hospital, Tongji Medical College, Huazhong \\ University of Science and Technology, Wuhan, China \\ Contributions: (I) Conception and design: Q Guo, HS Liu; (II) Administrative support: JL Guo, Y Yuan; (III) Provision of study materials or patients: \\ YQ Zhang; (IV) Collection and assembly of data: Y Yuan; (V) Data analysis and interpretation: J Zhang, CY Lin; (VI) Manuscript writing: All \\ authors; (VII) Final approval of manuscript: All authors. \\ \#These authors contributed equally to this work. \\ Correspondence to: Qiang Guo; Hua-Song Liu. Department of Cardiothoracic Surgery, Taihe Hospital, Hubei University of Medicine, Shiyan 442012, \\ China. Email: guoqianglidan@163.com; huasong819@qq.com.
}

Background: Targeted programmed cell death protein 1 (PD-1) therapy could effectively improve the long-term prognosis of patients with non-small cell lung cancer (NSCLC). The role of PD-1 targets in the progression of NSCLC has not been fully revealed.

Methods: The differentially expressed genes (DEGs) in patients' blood after NSCLC treatment with PD-1 blocker nivolumab in the GSE141479 dataset were analyzed by GEO2R and identified in the TCGA database. The mechanism of action involved in the PD-1 target molecules via the Gene Ontology (GO) and Kyoto Encyclopedia of Genes and Genomes (KEGG). The protein-protein interaction (PPI) network shows the relationship between PD-1 target molecules. The factors affecting the prognosis of NSCLC patients were identified via the COX regression analysis and survival analysis to build the risk model and nomogram. Results: There were 64 DEGs in patients' blood after nivolumab treatment and 48 DEGs in NSCLC tissues. The PD-1 target molecules involved cell proliferation, DNA replication, cell cycle, lung cancer, and other cellular processes. The prognostic factors CCNA2, CHEK1, DLGAP5, E2F8, FOXM1, HIST1H2BH, HJURP, MKI67, PLK1, TPX2, and TYMS, and the independent factors HIST1H2BH and PLK1, influenced the prognosis of NSCLC patients. HIST1H2BH and PLK1 were overexpressed in LUAD and LUSC tissues. The elevated expression levels of HIST1H2BH and PLK1 were related to the overall survival (OS) and the progression-free survival of NSCLC patients. High-risk NSCLC patients had a poor prognosis and were an independent factor influencing the poor prognosis of NSCLC patients. The high-risk model group was enriched with signaling mechanisms such as cell cycle, DNA replication, and homologous recombination.

Conclusions: The risk model based on PD-1 target molecules was helpful to assess the prognosis of NSCLC patients. HIST1H2BH and PLK1 might become prognostic biomarkers of NSCLC patients.

Keywords: Programmed cell death protein 1 (PD-1); non-small cell lung cancer (NSCLC); nivolumab

Submitted May 25, 2021. Accepted for publication Jul 08, 2021.

doi: 10.21037/atm-21-2963

View this article at: https://dx.doi.org/10.21037/atm-21-2963 


\section{Introduction}

In recent years, programmed cell death protein 1 (PD-1) has been one of the inhibitory immune checkpoints that activate T cells, NK cells, and B cells. PD-1 interacts with programmed death ligand 1 (PD-L1) to produce inhibitory signaling transduction, resulting in anti-tumor effects during cancer treatment (1). For example, in MKN45 and MGC-803 cells of gastric cancer (GC), anticytotoxic T lymphocyte antigen-4 (anti-CTLA-4) and anti-PD-1 combined therapy could significantly inhibit cell proliferation, migration, invasion, and epithelialmesenchymal transition (EMT), as well as induce cell apoptosis in vitro, and inhibit tumor formation in vivo. Mechanism studies have shown that the anti-CTLA-4 and anti-PD-1 combined therapy could inhibit the activation of $\beta$-catenin, MAPK, and PI3K/AKT signaling pathways. Interference with PD-1 expression inhibited GC cell proliferation, migration, invasion, and EMT and induced cell apoptosis (2). Inhibition of BET protein expression inhibited the expression level of PD-1/PD-L1 in triplenegative breast cancer. BET protein regulated the PD-1 expression level, reduced the production and signaling transduction of interferon- $\gamma$ in the $\mathrm{T}$ cells, and inhibited the expression of PD-L1 in breast cancer cells. Inhibition of BET protein improved tumor cell-specific T cell toxicity (3). In non-small cell lung cancer (NSCLC) tissues, PD-L1 expressionwas positive in $32.6 \%$ of patients. In patients with high-grade malignancy and lymph node metastasis, the expression level of PD-L1 increased significantly (4). In lung adenocarcinoma (LUAD), patients with low PDL1 expression levels had a longer overall survival (OS) (4). Cyclin-dependent kinase 7 (CDK7) mRNA and protein expression levels were related to the poor prognosis of NSCLC patients. The decrease of CDK7 expression level could cause NSCLC cell apoptosis and inhibit tumor growth. The CDK7 inhibitor THZ1 could down-regulate PD-L1 expression level by inhibiting MYC activity. THZ1 enhanced anti-tumor immunity by recruiting CD8 $\mathrm{T}$ cell infiltration and synergistically with anti-PD-1 therapy (5). This indicated that the anti-PD-1 treatment of cancer patients had importantvalue.

Nivolumab is the PD-1 inhibitor that binds to PD-L1 to the surface of $\mathrm{T}$ cells to block the immunosuppressive signaling pathway triggered by PDL-1/2 and restore the anti-tumor function of T cells. Currently, nivolumab is used to treatment the patients with unresectable or metastatic melanoma and advanced lung squamous cell carcinoma
(LUSC) and show the safe and effective therapeutic effect (6-9). For example, compared with the cytotoxic T-cell antigen-4 (CTLA-4) blocker ipilimumab, nivolumab has sustained recurrence-free survival benefits in patients with stage IIIB-C or IV melanoma after surgery. Nivolumab is also an effective adjuvant treatment for patients with high-risk melanoma after surgery (6). At present, many studies had shown that the PD-1 inhibitor nivolumab could improve the prognosis of cancer patients $(8,10,11)$. For example, Fujimoto et al. reported that the 6-month PFS rate of nivolumab in patients with advanced NSCLC was $56 \%$, the remission rate was $39 \%$, and the disease control rate was $72 \%$. There were no treatment-related deaths (8). Sugawara et al. reported that the median PFS of the nivolumab group was higher than that of the placebo group. Nivolumab treatment improved the PFS and objective remission rate of patients with advanced non-squamous NSCLC (10). Hoffner et al. reported that the combination of anti-PD-L1 immunotherapy and platinum-based chemotherapy can improve the prognosis of NSCLC and SCLC patients (11). Nivolumab has been effective therapeutic value in NSCLC treatment, and the mechanisms in anti-NSCLC treatment have not been fully revealed. Therefore, differentially expressed genes (DEGs) in the blood of patients from the GSE141479 dataset were analyzed after nivolumab treatment (12), the mechanisms of PD-1 target molecules in the progression of NSCLC were explored, and hub target molecules were screened in order to construct a risk model and nomogram to assess the prognosis of NSCLC patients. This could provide a theoretical reference point and new approach for cancer treatment.

We present the following article in accordance with the REMARK reporting checklist (available at https://dx.doi. org/10.21037/atm-21-2963).

\section{Methods}

The study was conducted in accordance with the Declaration of Helsinki (as revised in 2013).

\section{GSE141479 dataset}

The GSE141479 dataset of the Gene Expression Omnibus [GEO (http://www.ncbi.nlm.nih.gov/geo/)] database contained gene expression data from the blood samples of 74 NSCLC patients. Among them, 33 NSCLC patients were the blood samples treated with PD-1 inhibitor nivolumab. The platform information was GPL23126 
[Clariom_D_Human] Affymetrix Human Clariom D Assay [transcript (gene) version]. The gene expression levels were explored in blood samples of patients with NSCLC after nivolumab treatment via the GEO2R. Screening criteria: $\log \mathrm{FC}>0.585$ or $\log \mathrm{FC}<-0.585$ and $\mathrm{P}<0.05$.

\section{The Cancer Genome Atlas (TCGA) database}

The gene expression data of 108 normal lung tissue samples and 1,037 NSCLC tissue samples, the prognosis and clinicopathological characteristics data of NSCLC patients were downloaded from the TCGA (https://portal.gdc. cancer.gov/projects/) website. The limma package was used to analyze the expression levels of PD-1 target molecules in normal lung tissue and cancer tissue $(\log \mathrm{FC}>1$ or $\log \mathrm{FC}$ $<-1$ and $\mathrm{P}<0.05)$.

\section{DAVID database}

The DAVID database could analyze biological processes (BP), molecular functions (MF), cellular components (CC), signaling mechanisms, and the transcription factor regulation involved in multiple genes. In the DAVID database, the gene ontology (GO), Kyoto encyclopedia of genes and genomes (KEGG), and transcription factor analysis were used to analyze the MF, BP, CC, and signaling mechanisms involved in the abnormally expressed PD-1 target molecules of the TCGA database.

\section{Protein-protein interaction (PPI) network}

The abnormally expressed PD-1 target molecules in the TCGA database were entered into the Search Tool for the Retrieval of Interacting Genes (STRING) database to explore the PPI. The combination score $>0.4$ was the screening criterion. Cytoscape 3.6.1 software was used to visualize the relationship between proteins in the PPI network, and the MCODE method was used for enrichment analysis.

\section{The risk model and nomogram}

The prognostic datas of NSCLC in the TCGA database were sorted out, and missing values were deleted. PD-1 target gene expression and prognosis of NSCLC patients was spliced. Univariate Cox regression analysis evaluated the impact of PD-1 target molecules on the prognosis of NSCLC patients. Multivariate Cox regression analysis and the AIC method were used to screen independent factors affecting the prognosis of NSCLC patients, and a risk model was constructed. Kaplan-Meier (K-M) survival analysis and nomogram showed the adverse prognosis of NSCLC patients in the high- and low-risk groups.

\section{UALCAN database}

The expression levels of HIST1H2BH and PLK1 were explored in NSCLC subtypes LUAD and LUSC tissues by using the UALCAN database. The relationship between the expression levels of HIST1H2BH and PLK1 and the clinicopathological characteristics of LUAD and LUSC patients were explored via correlation analysis. Screening criteria: $\mathrm{P}<0.05$.

\section{K-M Plotter database}

K-M Plotter database was a commonly used prognostic analysis database. This database contained prognostic information for patients with lung cancer, breast cancer, and stomach cancer. In the K-M Plotter database, the median value of HIST1H2BH and PLK1 expression levels were grouped to evaluate their potential role in the prognosis of NSCLC patients.

\section{CbioPortal database}

The CbioPortal database provided large-scale cancer genomics datasets and visual analysis functions. In the CbioPortal database, the TCGA NSCLC data was used to show the mutations of HIST1H2BH and PLK1 in NSCLC and its subtypes LUAD and LUSC.

\section{Lung cancer explore (LCE) database}

The LCE database covered lung cancer gene expression data and prognostic information, and meta-analysis could be used to visualize gene expression in cancer patient tissues and its prognostic value. In the LCE database, the expression and prognostic value of HIST1H2BH and PLK1 were explored in lung cancer and its subtypes LUAD and LUSC via the meta-analysis.

\section{GEPIA database}

The data in the GEPIA database came from the TCGA and GTEx databases. Correlation analysis was used to explore 
the relationship between the expression level of PD-1 and the expression levels of risk model factors in normal lung tissues, LUAD, and LUSC tissues.

\section{The signaling mechanisms of the risk model}

Gene Set Enrichment Analysis (GSEA) was commonly used to evaluate the signaling mechanisms involved in influencing factors. In this study, we divided the TCGA NSCLC gene expression data into high- and low-risk groups based on the median value of risk scores, and the signaling mechanisms involved in the high- and low-risk groups were explored via the GSEA (13). The program was run for 1,000 cycles, and nominal $\mathrm{P}$ value $(\mathrm{NOM} \mathrm{P})<0.05$ was used as the screening criterion.

\section{Statistics analysis}

Blood of patients with NSCLC after nivolumab treatment was analyzed for DEGs using the GEO2R. The expression levels of PD-1 target molecules in NSCLC tissues were analyzed by the R limma package. Grouping criteria: median value of gene expression and risk score. Univariate and multivariate Cox regression analysis explored potential factors affecting the prognosis of NSCLC patients, including age, gender, clinical stage, T stage, lymph node metastasis, distant metastasis, high- and low-risk. K-M survival analysis showed the survival curve of NSCLC patients with high and low risk. $\mathrm{P}<0.05$ was considered statistically significant.

\section{Results}

\section{The expression levels of PD-1 target molecules in NSCLC}

Compared with 41 NSCLC patients, there were 64 DEGs in the blood of 33 NSCLC patients who received PD-1 inhibitor treatment $(\mathrm{P}<0.05)$. There are 11 highly expressed genes and 53 lowly expressed genes (Figure 1 and Table 1). In the TCGA database, compared with normal lung tissues, the expression levels of $48 \mathrm{PD}-1$ target molecules in NSCLC tissues were significantly increased (Figure 2 and Table 2).

\section{The biological functions and signaling mechanisms of PD-1 target molecules}

GO analysis showed that the PD-1 target molecules were involved in the mitotic nuclear division, chromosome segregation, cell division, cell proliferation, DNA binding, protein kinase binding, DNA replication, mitotic cytokinesis, DNA repair, and other cellular functions (Figure $3 A, B, C$ and Table $\mathrm{S} 1)$. KEGG signaling mechanism analysis showed that the PD-1 target molecules were involved in viral carcinogenesis, systemic lupus erythematosus, alcoholism, cell cycle, and progesterone-mediated oocyte maturation (Figure 3D). In addition, PD-1 target molecules were also involved in breast cancer, ovarian cancer, leukemia, bladder cancer, chronic obstructive pulmonary disease, esophageal adenocarcinoma, lung cancer, head and neck cancer, and other diseases (Figure S1 and Table 3).

\section{The network of PD-1 target molecules}

In the DAVID database, there were 3 transcription factors (NFY, E2F, and MEF2) enriched by 48 PD-1 target molecules $(\mathrm{P}<0.05)$. Figure $\mathrm{S} 2$ showed the relationship between transcription factors NFY, E2F, MEF2, and PD-1 target molecules, and the PPI network was used to visualize the relationship between the proteins of 48 PD-1 target molecules (Figure 4).

\section{Construction of PD-1 target molecular risk model}

Univariate Cox regression analysis showed that CCNA2, CHEK1, DLGAP5, E2F8, FOXM1, HIST1H2BH, HJURP, MKI67, PLK1, TPX2, and TYMS were the prognostic factors of NSCLC patients (Figure $5 A$ and Table 4). On this basis, multivariate Cox regression analysis and AIC screening were performed, and it was found that HIST1H2BH and PLK1 were independent factors influencing the prognosis of NSCLC patients (Figure $5 B$ and Table 5). Figure $5 C$ and $5 D$ showed the high and low scores and survival status of NSCLC patients.

\section{The value of risk model factors was verified in NSCLC}

In the UALCAN database, HIST1H2BH was overexpressed in NSCLC subtype LUAD tissues. The expression level of HIST1H2BH was correlated with the age, gender, smoking history, and histological subtype of LUAD patients (Figure $6 A$ and Table 6). PLK1 was overexpressed in LUAD tissues. The expression level of PLK1 was correlated with the age, gender, clinical stage, smoking history, histological subtype, and TP53 mutation status of LUAD patients (Figure $6 B$ and Table 6). HIST1H2BH was overexpressed in NSCLC subtype LUSC tissues. The expression level 
A GSE141479


Figure 1 The expression level of the PD-1 target molecules in GSE141479 NSCLC blood samples. (A) Box plot showing that the samples have good consistency; (B) the expression density of sample genes; (C) highly expressed genes; (D) lowly expressed genes. PD-1, programmed cell death protein 1; NSCLC, non-small cell lung cancer.

of HIST1H2BH was correlated with smoking history and histological subtypes in LUSC patients (Table 7). PLK1 was overexpressed in LUSC tissues. The expression level of PLK1 was related to the age, gender, clinical stage, smoking history, lymph node metastasis, and TP53 mutation status of LUSC patients (Table 7). In the K-M Plotter database, $\mathrm{K}-\mathrm{M}$ survival analysis showed that elevated expression levels of HIST1H2BH and PLK1 were related to the OS and PFS of NSCLC patients (Figure 7).

In addition, the meta-analysis results showed that HIST1H2B and PLK1 were overexpressed in lung cancer tissues, and the hazard ratio (HR) were 1.18 and 1.71 , respectively, and they were statistically significant (Figure $8 A, B)$. The overexpression of HIST1H2BH and PLK1 indicated that the prognosis of lung cancer patients was not better. The HR was 1.05 and 1.16. There was no statistical significance between the overexpression of HIST1H2BH and the prognosis of lung cancer patients (Figure 8C,D). The results of subgroup analysis showed that HIST1H2BH was overexpressed in LUAD and LUSC tissues, with HR of 1.27 and 2.1, respectively, which were statistically significant (Figure S3A). PLK1 was overexpressed in LUAD and LUSC tissues, and the HR was 1.79 and 3.15, respectively, which were statistically significant (Figure S3B). The overexpression of HIST1H2BH suggested that the prognosis of LUAD patients was poor, and the HR was 1.08 , which is statistically significant (Figure S4A). The low expression of HIST1H2BH indicated a poor prognosis for LUSC patients, and the HR was 0.89 , which was statistically significant (Figure S4A). The overexpression of PLK1 
Table 1 The expression level of PD-1 target molecules in GSE141479 NSCLC blood samples

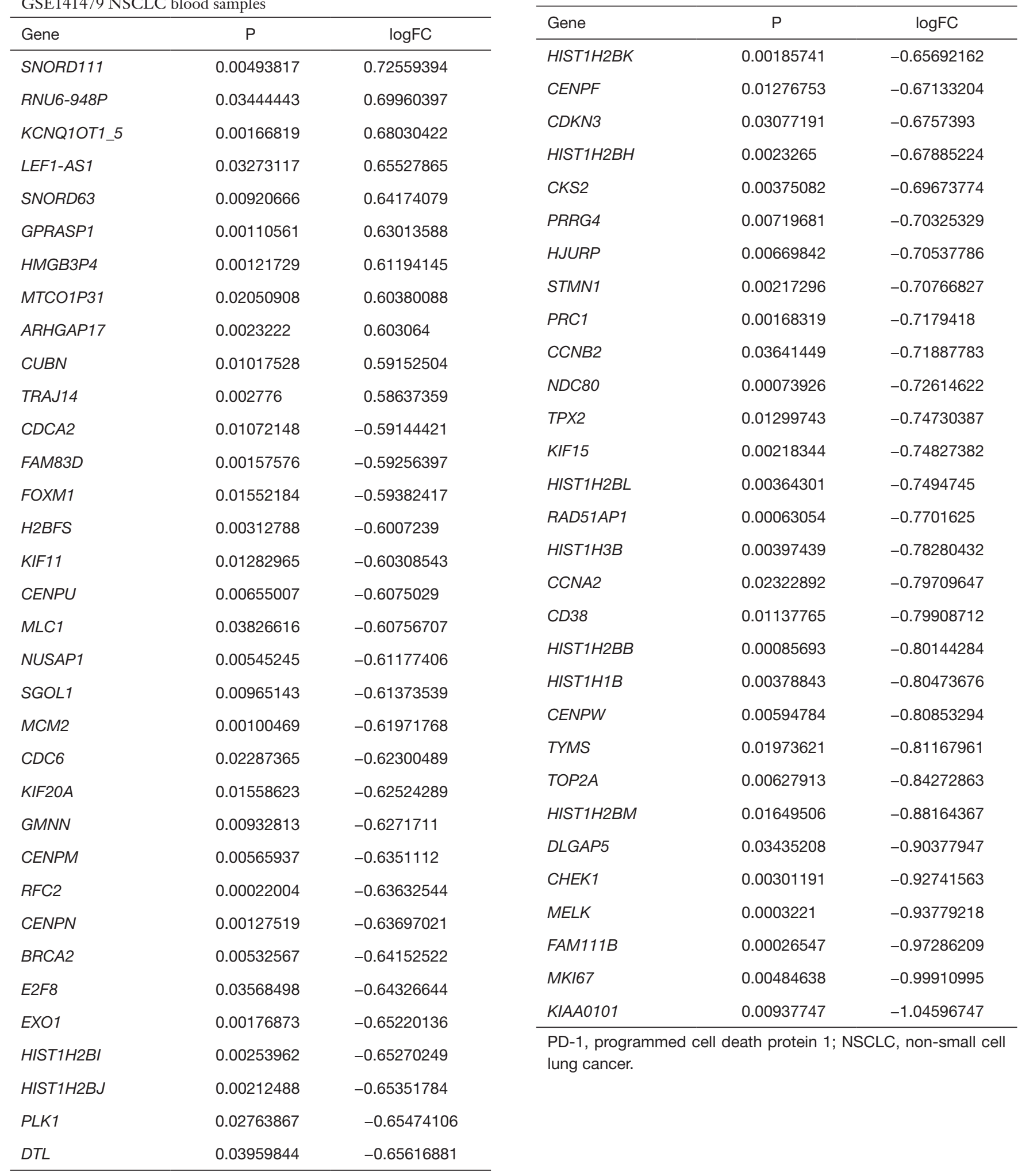

Table 1 (continued) 

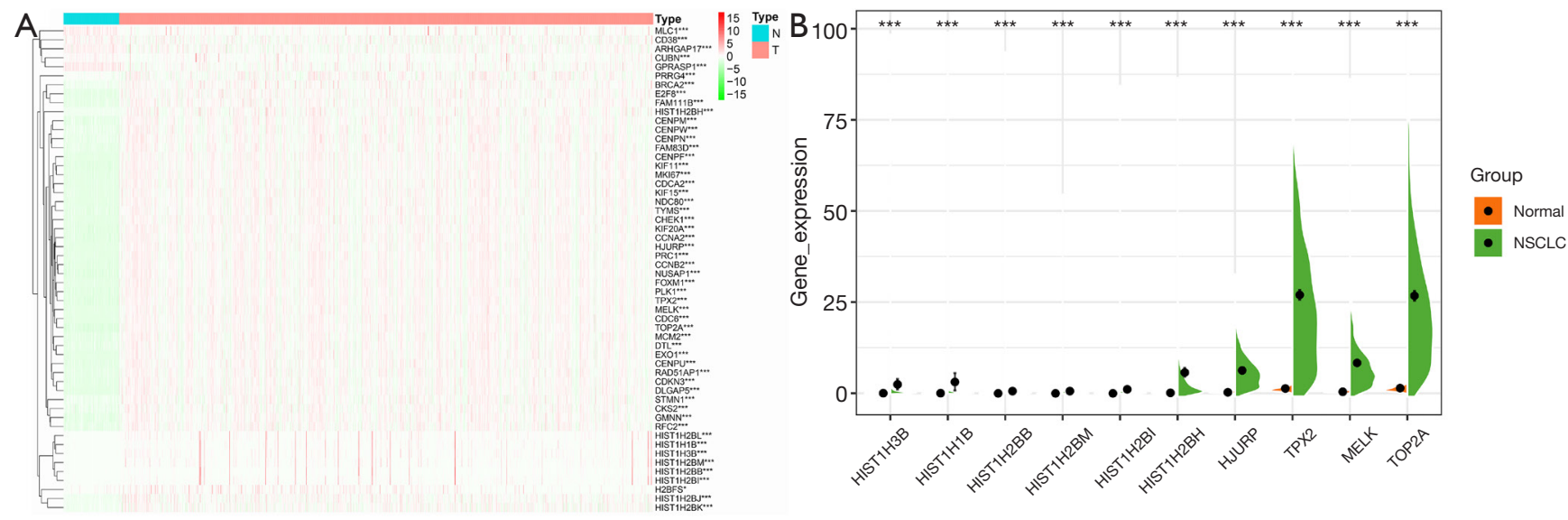

Figure 2 The expression level of PD-1 target molecules in the TCGA NSCLC tissues. (A) Heat map representing the DEGs; (B) violin chart demonstrating the top 10 DEGs via the fold-change. ${ }^{* * *}, \mathrm{P}<0.001$. PD-1, programmed cell death protein 1 ; TCGA, the cancer genome atlas; NSCLC, non-small cell lung cancer; DEGs, differentially expressed genes.

Table 2 The expression levels of PD-1 target molecules in NSCLC tissues

\begin{tabular}{|c|c|c|}
\hline Gene & $\log F C$ & $P$ \\
\hline HIST1H3B & 6.971563358 & $5.22 E-42$ \\
\hline HIST1H1B & 6.889830378 & $1.98 \mathrm{E}-34$ \\
\hline HIST1H2BB & 6.6176645 & 2.92E-16 \\
\hline HIST1H2BM & 6.546656387 & $1.70 \mathrm{E}-27$ \\
\hline HIST1H2BI & 6.144328211 & 3.81E-19 \\
\hline HIST1H2BH & 5.25752465 & 1.97E-39 \\
\hline HJURP & 4.423193962 & $9.76 \mathrm{E}-63$ \\
\hline TPX2 & 4.356893524 & 1.49E-61 \\
\hline MELK & 4.28293495 & 2.60E-61 \\
\hline TOP2A & 4.239513136 & 6.71E-63 \\
\hline FOXM1 & 4.206146682 & $1.40 \mathrm{E}-60$ \\
\hline EXO1 & 4.176032779 & 4.79E-62 \\
\hline DLGAP5 & 4.123032333 & $1.54 \mathrm{E}-61$ \\
\hline CENPF & 4.004101702 & 2.23E-61 \\
\hline CDC6 & 3.962775113 & 8.09E-62 \\
\hline PLK1 & 3.804871556 & 1.14E-62 \\
\hline CCNB2 & 3.791913823 & 1.42E-61 \\
\hline HIST1H2BL & 3.771763049 & 4.85E-33 \\
\hline NDC80 & 3.66052765 & $1.20 \mathrm{E}-60$ \\
\hline CDCA2 & 3.618670951 & 2.97E-60 \\
\hline KIF20A & 3.560206265 & $2.46 \mathrm{E}-61$ \\
\hline CCNA2 & 3.560063035 & $9.08 \mathrm{E}-61$ \\
\hline MKI67 & 3.512151918 & 2.23E-58 \\
\hline CDKN3 & 3.434927857 & $1.04 \mathrm{E}-57$ \\
\hline
\end{tabular}

Table 2 (continued)
Table 2 (continued)

\begin{tabular}{|c|c|c|}
\hline Gene & $\log F C$ & $P$ \\
\hline FAM83D & 3.412001161 & $2.99 E-50$ \\
\hline E2F8 & 3.334165686 & $2.05 \mathrm{E}-61$ \\
\hline PRC1 & 3.322365587 & $1.58 \mathrm{E}-61$ \\
\hline KIF11 & 3.262694147 & 7.05E-62 \\
\hline NUSAP1 & 3.233047006 & $1.10 \mathrm{E}-60$ \\
\hline KIF15 & 3.110763871 & $7.51 \mathrm{E}-59$ \\
\hline FAM111B & 3.110199706 & $4.99 \mathrm{E}-57$ \\
\hline MCM2 & 2.988886147 & $5.17 \mathrm{E}-58$ \\
\hline CENPU & 2.922017757 & 3.96E-59 \\
\hline$R A D 51 A P 1$ & 2.910137622 & $4.25 E-55$ \\
\hline CHEK1 & 2.741114388 & $2.08 \mathrm{E}-61$ \\
\hline HIST1H2BJ & 2.624552264 & $7.72 \mathrm{E}-28$ \\
\hline CENPM & 2.594186936 & $9.10 \mathrm{E}-51$ \\
\hline CENPW & 2.583310716 & $1.63 E-42$ \\
\hline$D T L$ & 2.466385804 & $3.72 \mathrm{E}-50$ \\
\hline TYMS & 2.401391345 & $4.02 E-56$ \\
\hline GMNN & 1.900079663 & $1.15 E-56$ \\
\hline CENPN & 1.899039582 & $1.93 \mathrm{E}-47$ \\
\hline STMN1 & 1.625279048 & 2.36E-35 \\
\hline CKS2 & 1.53273206 & $6.79 \mathrm{E}-34$ \\
\hline BRCA2 & 1.461967704 & $6.31 \mathrm{E}-37$ \\
\hline HIST1H2BK & 1.422823205 & 4.16E-26 \\
\hline RFC2 & 1.239427886 & $1.96 \mathrm{E}-48$ \\
\hline H2BFS & 1.214789768 & 0.02134121 \\
\hline
\end{tabular}

PD-1, programmed cell death protein 1; NSCLC, non-small cell lung cancer. 

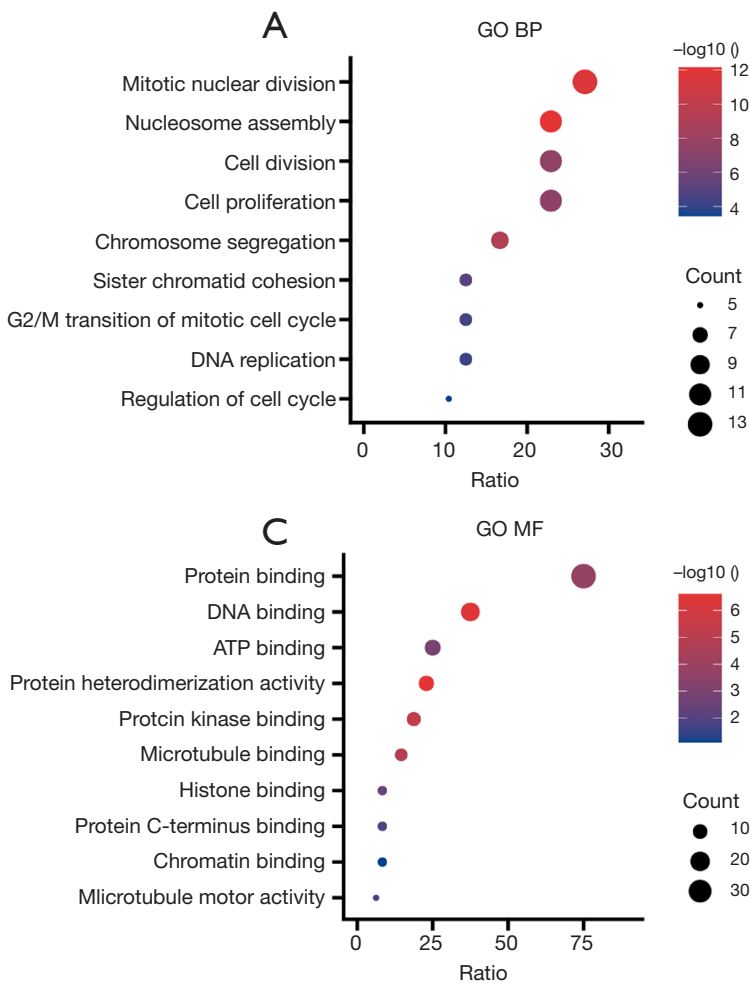
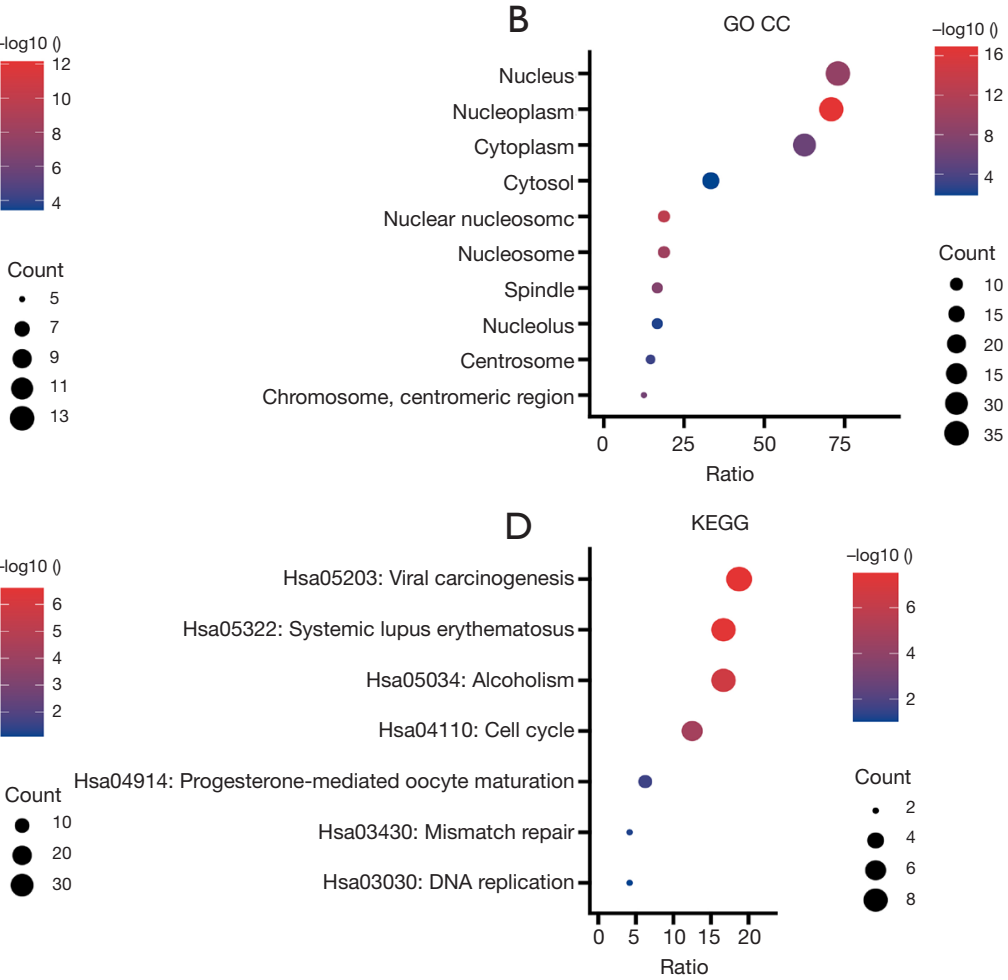

Figure 3 The biological functions and signaling mechanisms of PD-1 target molecules via the GO and KEGG. (A) BP; (B) CC; (C) MF; (D) KEGG. GO, gene ontology; BP, biological processes; CC, cellular component; MF, molecular function; KEGG, Kyoto encyclopedia of genes and genomes; PD-1, programmed cell death protein 1.

Table 3 Disease related to PD-1 target molecules

\begin{tabular}{|c|c|c|c|}
\hline Category & Term & Count & $P$ \\
\hline GAD_DISEASE & Breast Cancer & 10 & $2.72 \mathrm{E}-05$ \\
\hline GAD_DISEASE & Longevity & 9 & $1.68 \mathrm{E}-05$ \\
\hline GAD_DISEASE & Ovarian cancer & 7 & $4.88 \mathrm{E}-04$ \\
\hline GAD_DISEASE & Breast cancer & 7 & 0.002764474 \\
\hline GAD_DISEASE & Chronic obstructive pulmonary disease & 6 & 0.005391657 \\
\hline GAD_DISEASE & Lung cancer & 6 & 0.013295264 \\
\hline GAD_DISEASE & Lung Cancer & 6 & 0.022918521 \\
\hline GAD_DISEASE & Leukemia, Lymphocytic, Chronic, B-Cell & 5 & 0.002712277 \\
\hline GAD_DISEASE & Adenocarcinoma|Pancreatic Neoplasms & 3 & 0.009443208 \\
\hline GAD_DISEASE & Head and neck cancer & 3 & 0.026277742 \\
\hline GAD_DISEASE & Abortion, Spontaneous & 3 & 0.036618707 \\
\hline
\end{tabular}

PD-1, programmed cell death protein 1. 


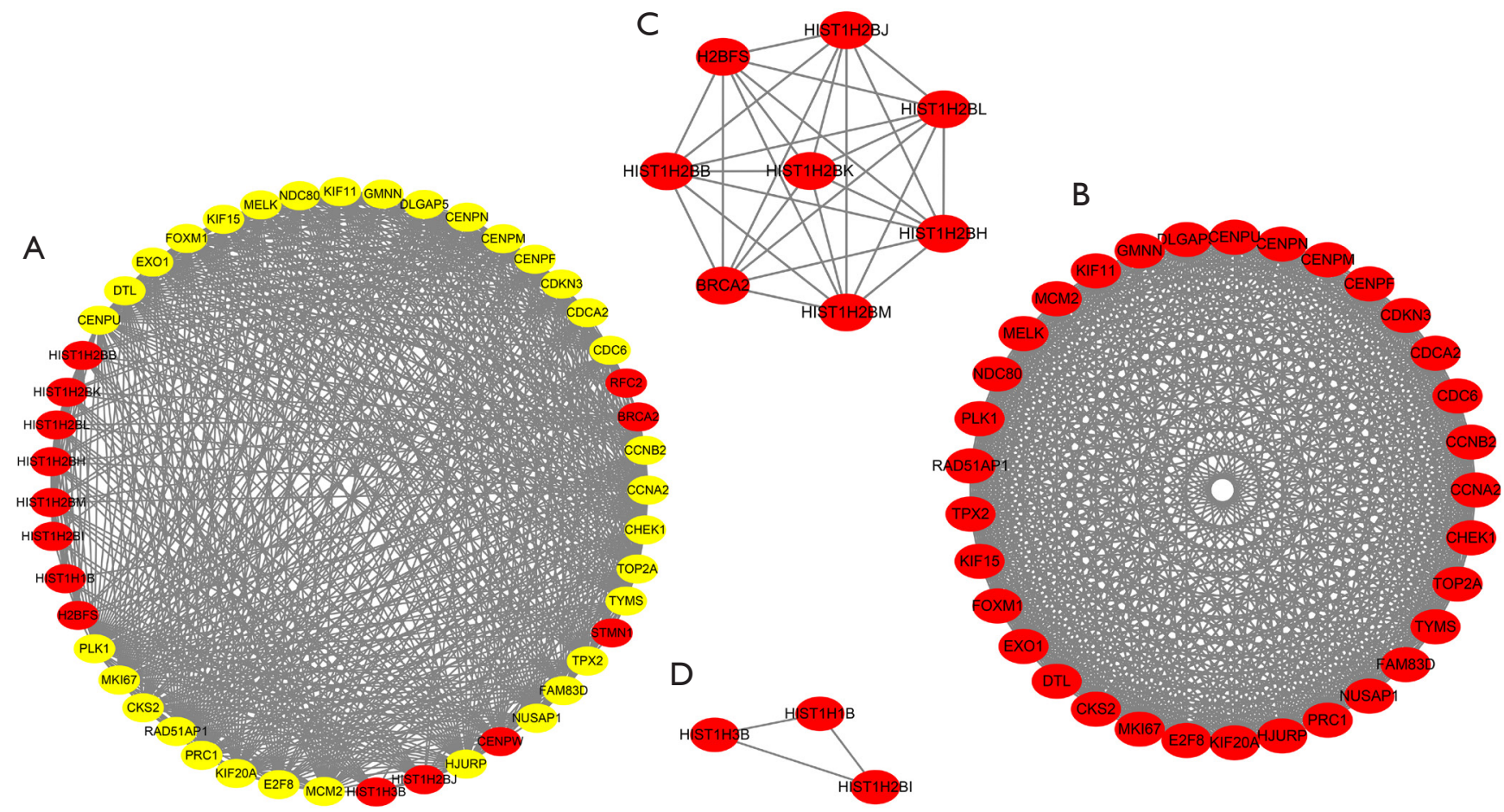

Figure 4 The PPI network of 48 PD-1 target molecules. (A) PPI network; (B,C,D) the enrichment analysis by MCODE. PPI, proteinprotein interaction; PD-1, programmed cell death protein 1.

A

$\begin{array}{lrr} & \text { pvalue } & \begin{array}{r}\text { Hazard ratio } \\ \text { CCNA2 }\end{array} \\ 0.024 & 1.119(1.015-1.233) \\ \text { CHEK1 } & 0.045 & 1.161(1.003-1.344) \\ \text { DLGAP5 } & 0.036 & 1.120(1.007-1.246) \\ \text { E2F8 } & 0.031 & 1.188(1.015-1.391) \\ \text { FOXM1 } & 0.018 & 1.106(1.017-1.202) \\ \text { HIST1H2BH } & 0.008 & 0.897(0.827-0.972) \\ \text { HJURP } & 0.046 & 1.113(1.002-1.237) \\ \text { MKI67 } & 0.034 & 1.118(1.008-1.240) \\ \text { PLK1 } & 0.003 & 1.185(1.061-1.323) \\ \text { TPX2 } & 0.022 & 1.102(1.014-1.197) \\ \text { TYMS } & 0.016 & 1.147(1.026-1.282)\end{array}$

B

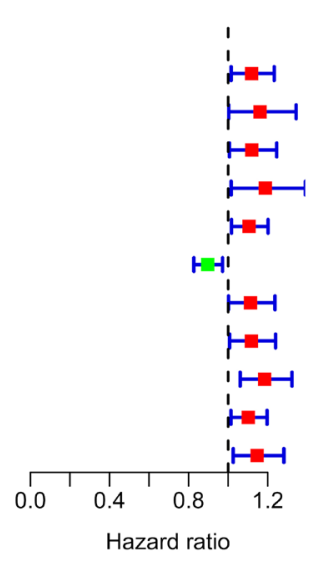

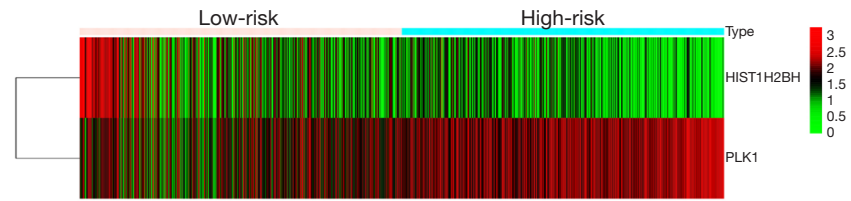

C
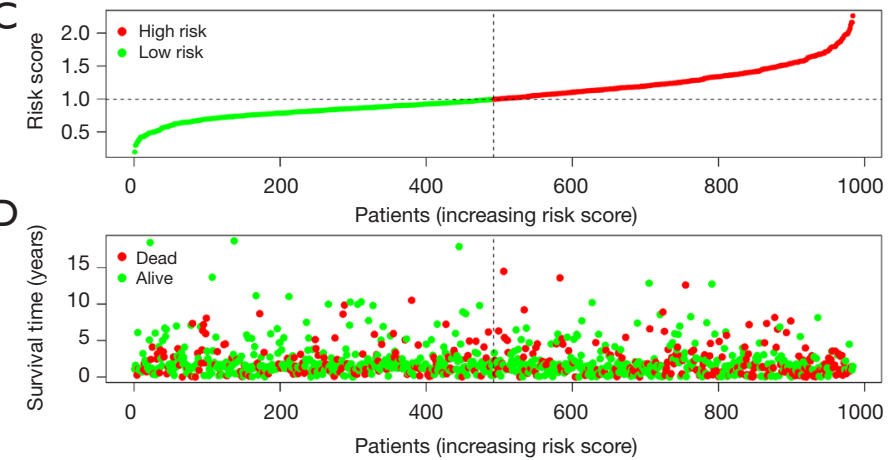

Figure 5 The risk model of PD-1 target molecules. (A) Univariate Cox regression analysis of the prognostic factors; (B) heat map showing the relationship between risk and prognostic factors; (C,D) the risk scores and survival status of NSCLC patients. PD-1, programmed cell death protein 1; NSCLC, non-small cell lung cancer. 
Table 4 Univariate Cox regression analysis showed the prognostic factors of NSCLC patients

\begin{tabular}{lcccc}
\hline Gene & HR & HR.95L & HR.95H & P \\
\hline CCNA2 & 1.119030214 & 1.015250316 & 1.233418597 & 0.023527732 \\
CHEK1 & 1.161268534 & 1.003467151 & 1.343885155 & 0.044812367 \\
DLGAP5 & 1.120187116 & 1.007468832 & 1.24551662 & 0.035951079 \\
E2F8 & 1.188481809 & 1.015445446 & 1.391004328 & 0.031485368 \\
FOXM1 & 1.105655354 & 1.017333811 & 1.20164468 & 0.018052236 \\
HIST1H2BH & 0.896796742 & 0.827448404 & 0.971957155 & 0.007986484 \\
HJURP & 1.113339724 & 1.001826182 & 1.237265867 & 0.046168795 \\
MKI67 & 1.118363726 & 1.008411159 & 1.24030502 & 0.034124878 \\
PLK1 & 1.184761851 & 1.061018977 & 1.322936416 & 0.002592543 \\
TPX2 & 1.102047152 & 1.014411384 & 1.19725384 & 0.021538305 \\
TYMS & 1.146977658 & 1.026076189 & 0.015825538 & \\
\hline
\end{tabular}

NSCLC, non-small cell lung cancer.

Table 5 The independent prognostic factors of NSCLC patients

\begin{tabular}{|c|c|c|c|c|c|}
\hline Gene & coef & $\mathrm{HR}$ & HR.95L & HR.95H & $\mathrm{P}$ \\
\hline PLK1 & 0.303398284 & 1.354453815 & 1.199191723 & 1.529818043 & $1.04 \mathrm{E}-06$ \\
\hline
\end{tabular}

NSCLC, non-small cell lung cancer.

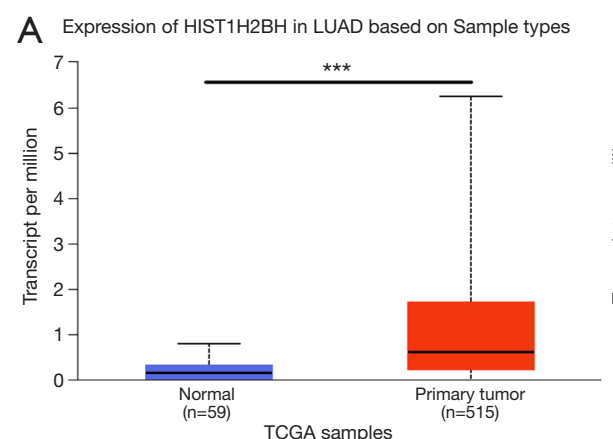

B Expression of PLK1 in LUAD based on Sample types

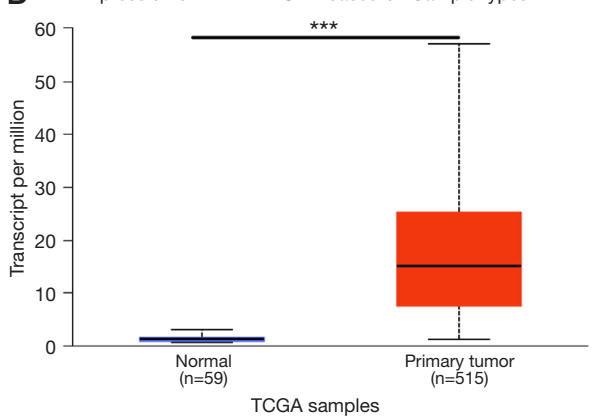

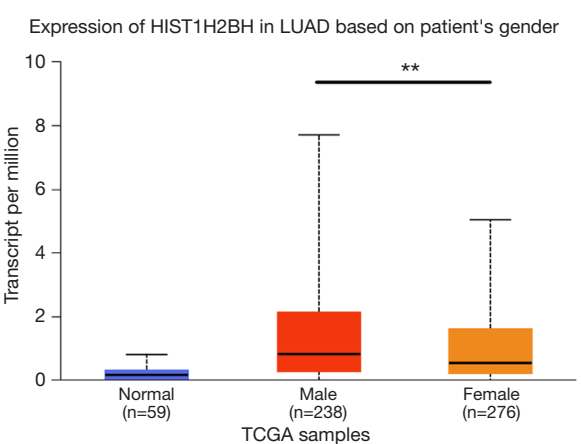

Expression of PLK1 in LUAD based on patient's gender





Expression of PLK1 in LUAD based on patient's age

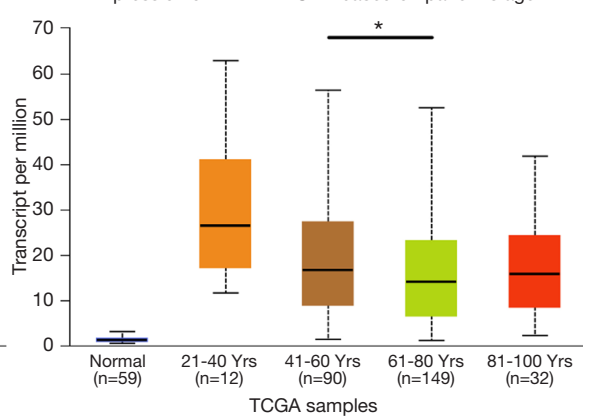

Figure 6 The overexpression levels of HIST1H2BH and PLK1 were correlated with the gender and smoking history of LUAD patients in the UALCAN database. (A) HIST1H2BH; (B) PLK1. *, $\mathrm{P}<0.05$ **, $\mathrm{P}<0.01$; **, $\mathrm{P}<0.001$. LUAD, lung adenocarcinoma. 
Table 6 The value of risk model factors in LUAD

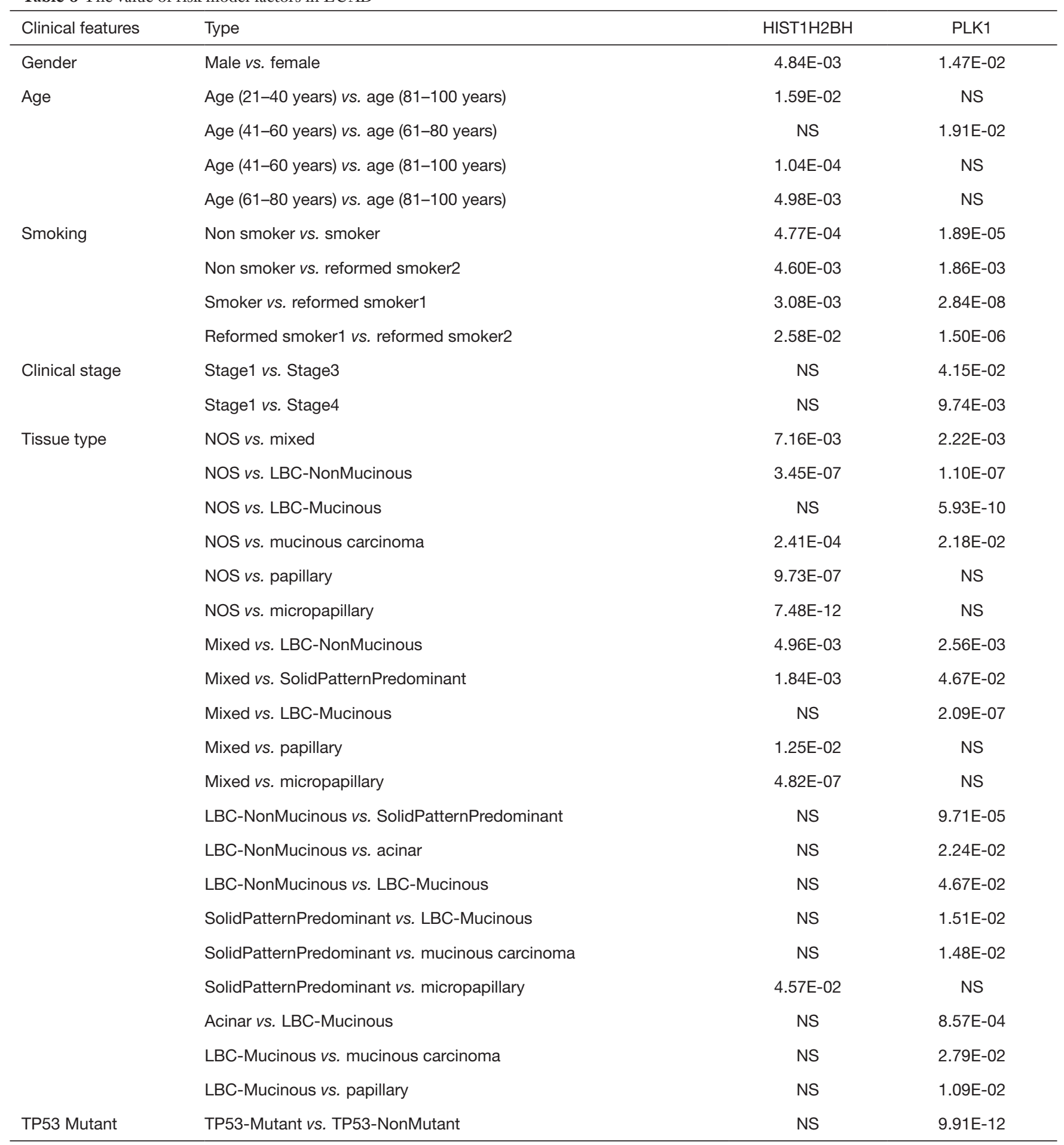

LUAD, lung adenocarcinoma. 
Table 7 The value of risk model factors in LUSC

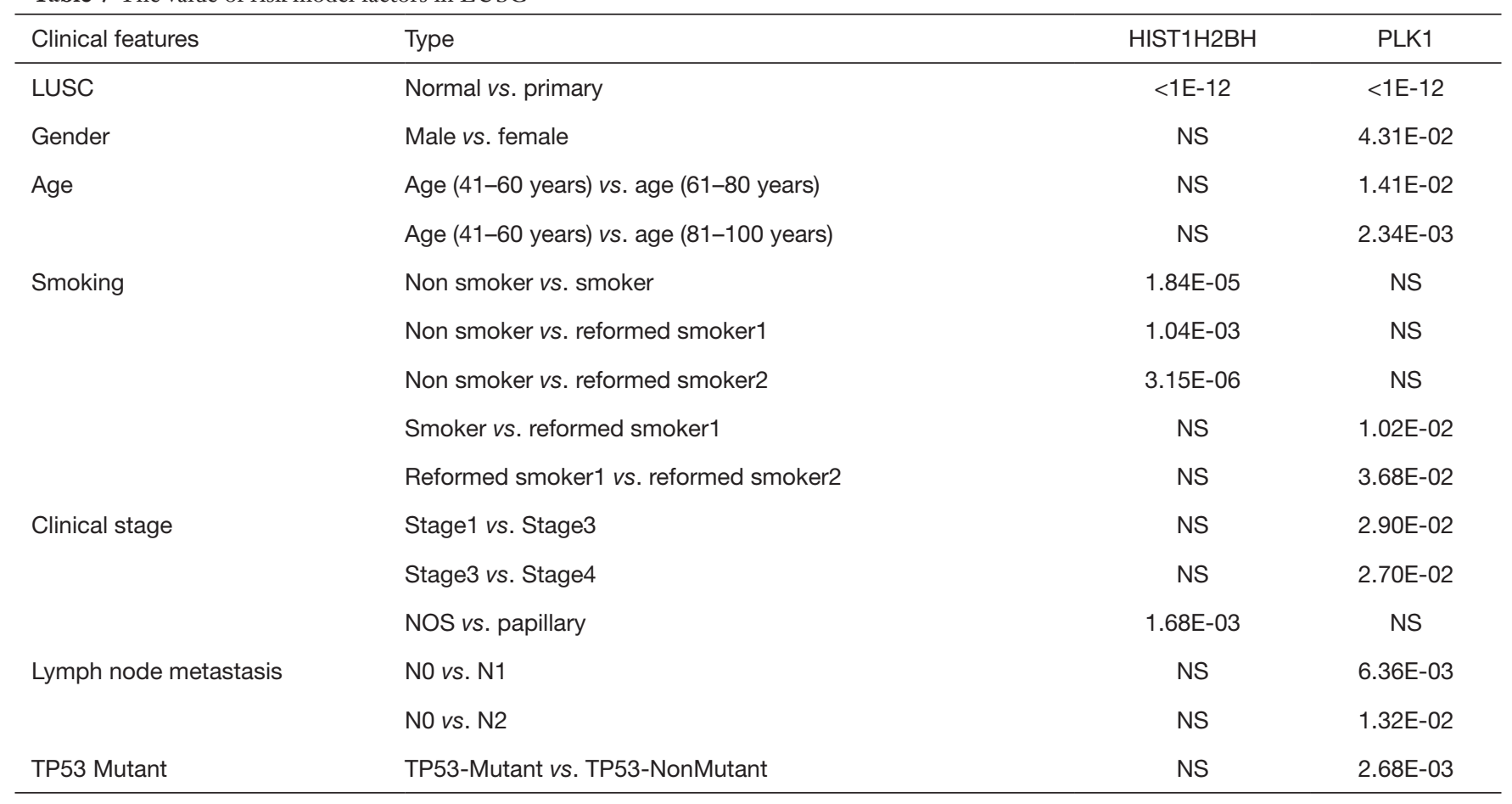

LUSC, lung squamous cell carcinoma.

suggested a poor prognosis for LUAD and LUSC patients, and the HR was 1.21 and 1.05, respectively. However, there was no statistical significance between the overexpression of PLK1 and the prognosis of LUSC patients (Figure S4B). In addition, we also found that HIST1H2BH and PLK1 had significant mutations in NSCLC (Figure S5).

\section{Evaluation of the risk model in the prognosis of NSCLC patients}

K-M survival analysis showed that high-risk NSCLC patients in the risk model had poor prognoses (Figure 9A). Univariate Cox regression analysis showed that the clinical stage, $\mathrm{T}$ stage, lymph node metastasis, and risk score were the influencing factors for the poor prognosis of NSCLC patients (Figure 9B). Multivariate Cox regression analysis showed that the clinical stage, $\mathrm{T}$ stage, and risk score were independent factors influencing the poor prognosis of NSCLC patients (Figure 9C). The nomogram showed that the clinical stage was the biggest influence on the survival of NSCLC patients, and the prognosis of NSCLC patients became worse as the stage increased. The second biggest influence was the risk score. As the risk score increased, the prognosis of NSCLC patients became worse (Figure 10).

\section{The signaling mechanisms involved in the risk model}

The high-risk group had significant roles in the cell cycle, mismatch repair, progesterone mediated oocyte maturation, oocyte meiosis, DNA replication, homologous recombination, ubiquitin-mediated proteolysis, proteasome, base excision repair, basal transcription factors, RNA degradation, p53 signaling pathway, glyoxylate and dicarboxylate metabolism, citrate cycle TCA cycle, lysine degradation, purine metabolism, NSCLC, and other cell types and functions (Figure 11 and Table 8).

\section{The expression levels of $\mathrm{HIST} 1 \mathrm{H} 2 \mathrm{BH}$ and $\mathrm{PLK} 1$ were correlated with the expression level of $P D-1$}

In lung tissue, the expression level of PLK1 was significantly correlated with the expression level of PD-1 (Figure S6A). Subgroup analysis showed that the expression level of PLK1 in normal tissues derived from LUAD and LUSC was significantly correlated with the expression level of PD-1 (Figure S6B,C). In LUAD and LUSC tissues, the expression level of PLK1 was significantly correlated with the expression level of PD-1 (Figure S6D,E). In lung tissue, the expression level of HIST1H2BH was significantly 

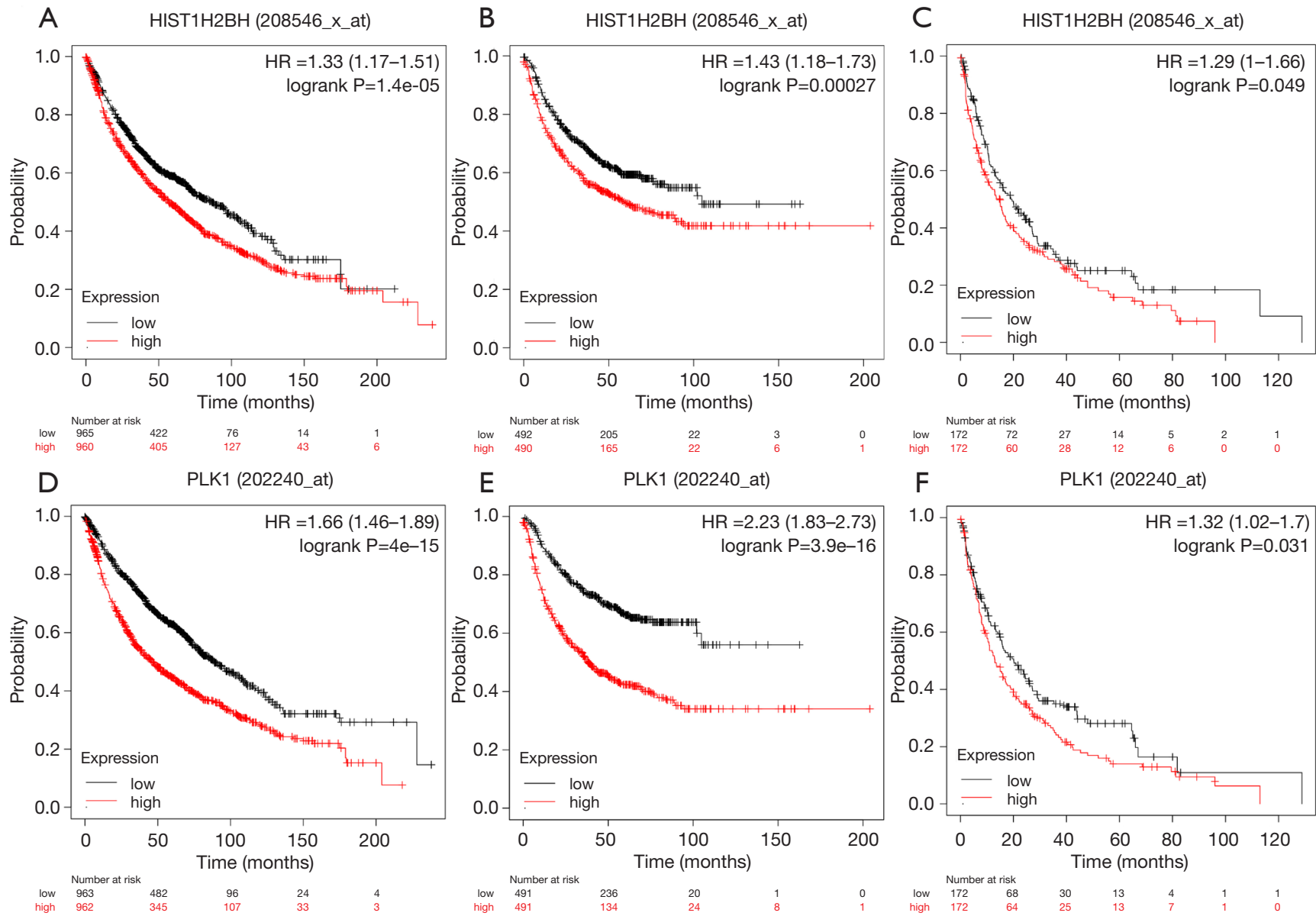

PLK1 (202240_at)

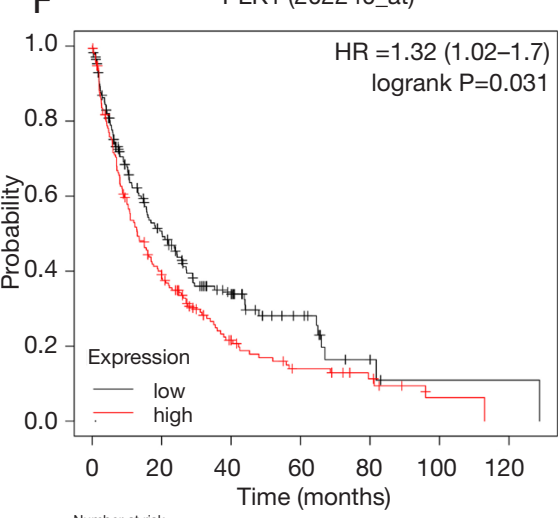

Figure 7 Elevated expression levels of HIST1H2BH and PLK1 were related to the prognosis of NSCLC patients. (A,B,C) The OS, FP, and PPS of HIST1H2BH overexpression level in NSCLC patients; (D,E,F) the OS, FP, and PPS of PLK1 overexpression level in NSCLC patients. NSCLC, non-small cell lung cancer; OS, overall survival; PF, progression-free survival; PPS, post-progression survival.

correlated with the expression level of PD-1 (Figure S6F). Subgroup analysis showed that the expression level of HIST1H2BH in normal tissues derived from LUSC was significantly correlated with the expression level of PD-1 (Figure S6G). In LUSC tissue, the expression level of HIST1H2BH was significantly correlated with the expression level of PD-1 (Figure S6H).

\section{Discussion}

Recent years, NSCLC was one of the top tumors in the world in terms of morbidity and mortality (14). There were a variety of treatments for NSCLC patients that could improve the prognosis of NSCLC patients. However, the treatment effect was still not satisfactory. In recent years, immunotherapy was considered to be one of the effective ways to improve advanced cancer. PD-1 inhibitor nivolumab could block the binding of PD-1 and PD-L1, promote T cells to participate in the recognition and killing of tumor cells, and prolong the survival of NSCLC patients (15). Therefore, this study explored the signaling mechanisms of PD-1 in the treatment of NSCLC and screen potential new target molecules for NSCLC progression to improve the long-term prognosis of NSCLC patients. In our current research, 48 PD-1 target molecules were involved in the cell division, cell proliferation, DNA replication, cell cycle, Lung Cancer, etc. studies indicated that cell proliferation, DNA replication, cell cycle played an important role in the development of NSCLC (16-18). For example, compared with paired normal lung tissues and bronchial epithelial cell, the expression level of TRIM13 in NSCLC tissues and cells was decreased. TRIM13 overexpression 
A

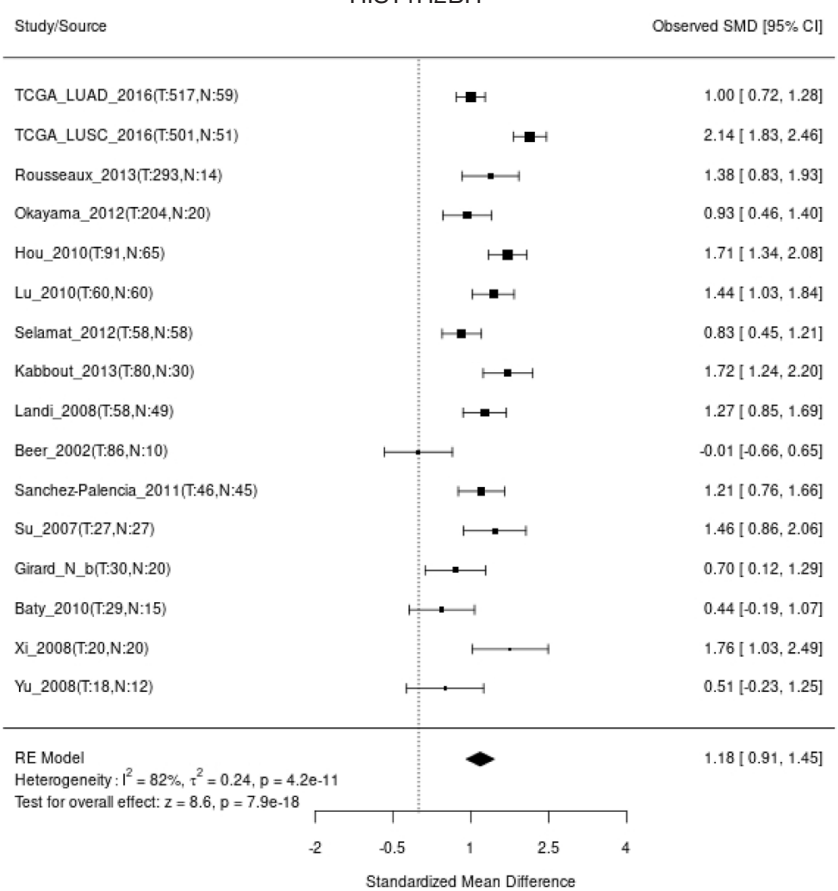

C

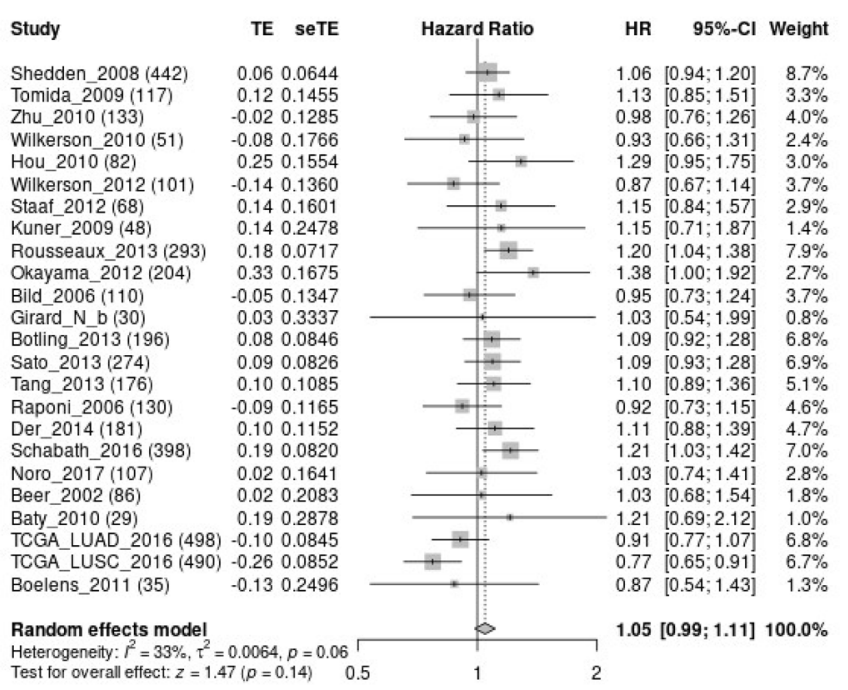

B

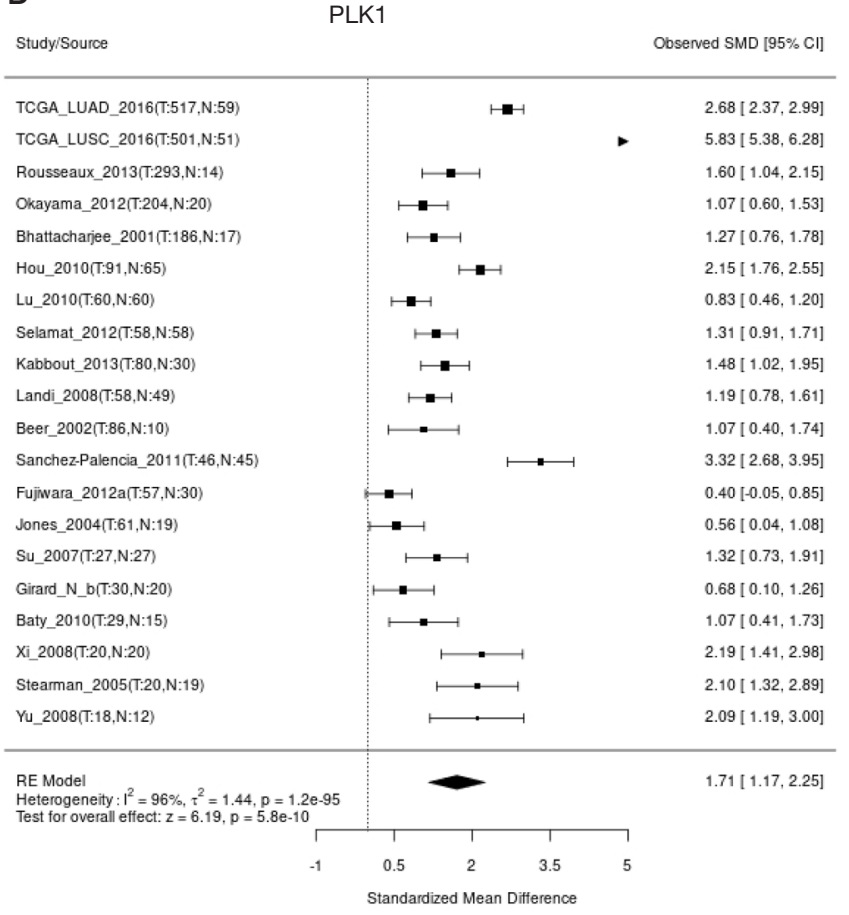

PLK1

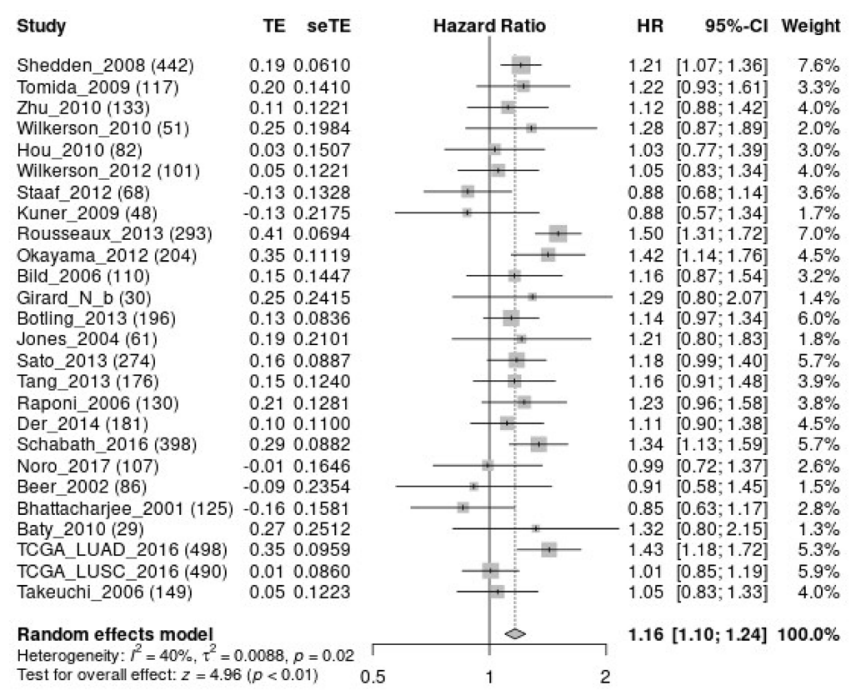

Figure 8 HIST1H2B and PLK1 overexpression levels were related to the prognosis of lung cancer via the meta-analysis. (A,B) HIST1H2B and PLK1 expression levels; (C,D) the prognosis values of HIST1H2B and PLK1. 


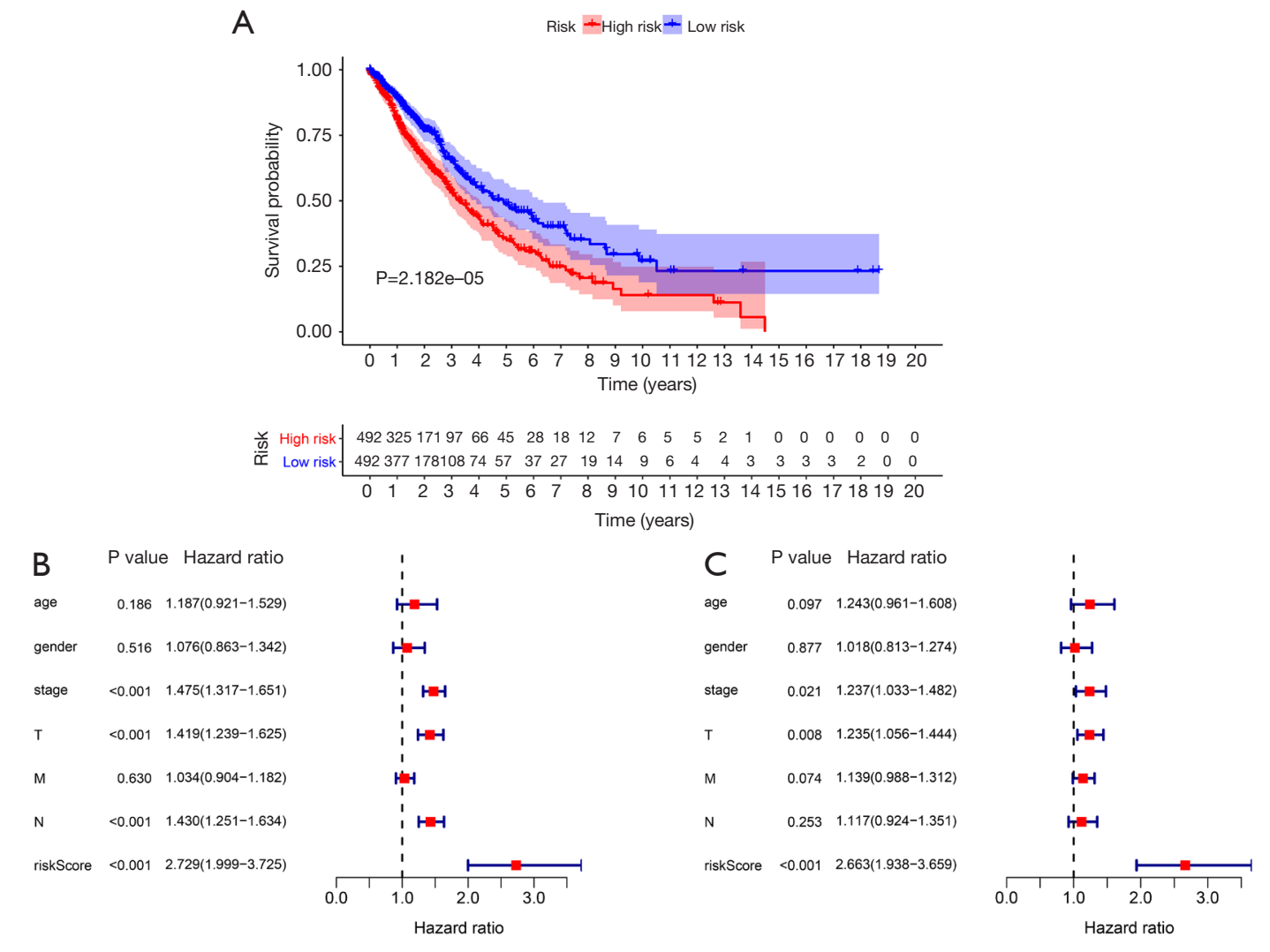

Figure 9 The risk model was related to the prognosis of NSCLC patients. (A) K-M survival analysis; (B) univariate Cox regression analysis; (C) multivariate Cox regression analysis. NSCLC, non-small cell lung cancer; K-M, Kaplan-Meier.

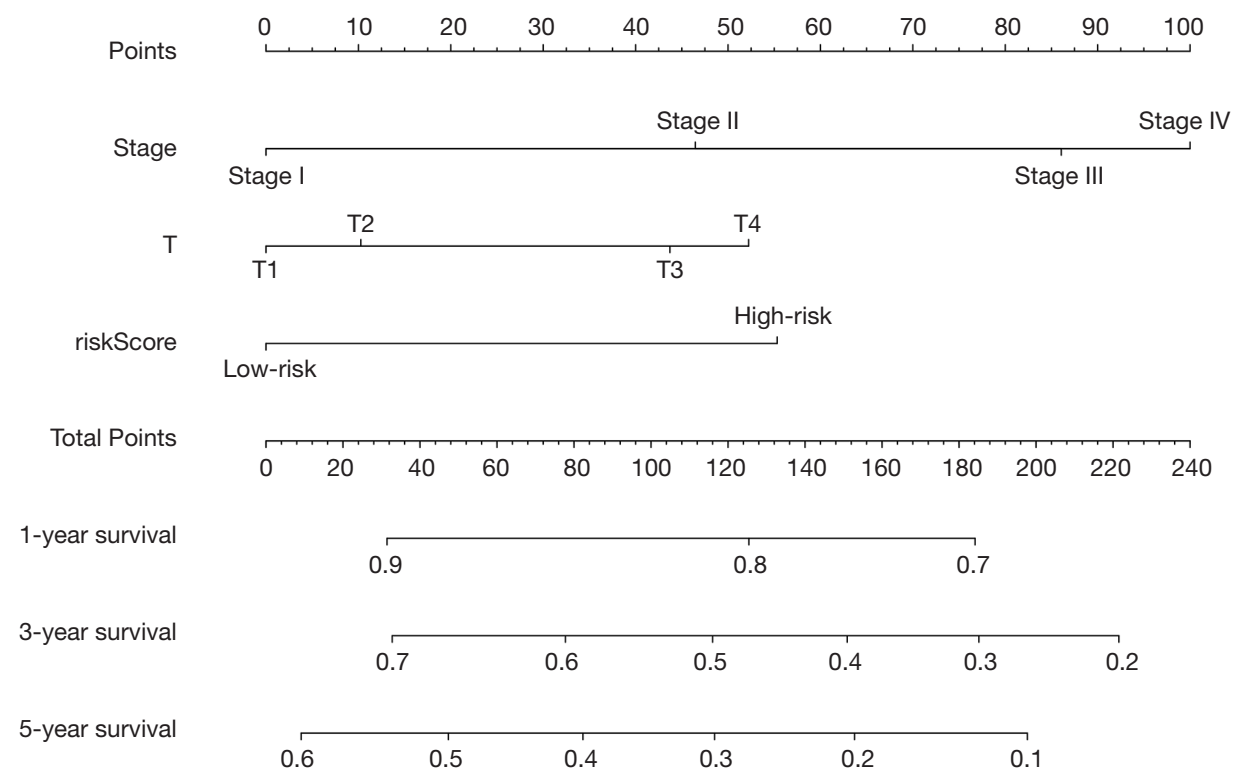

Figure 10 Construction of the risk model nomogram. 
A

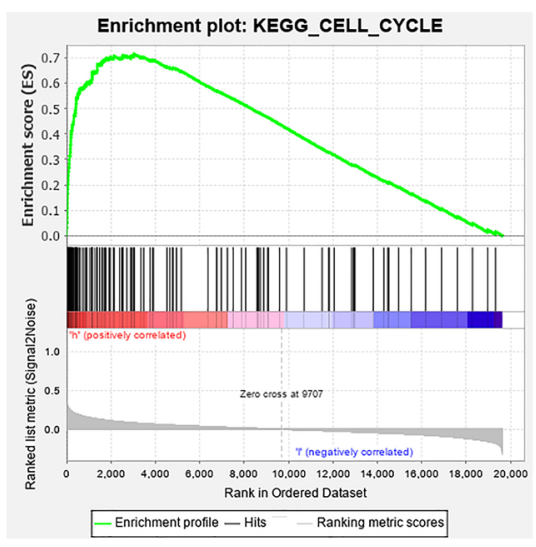

D

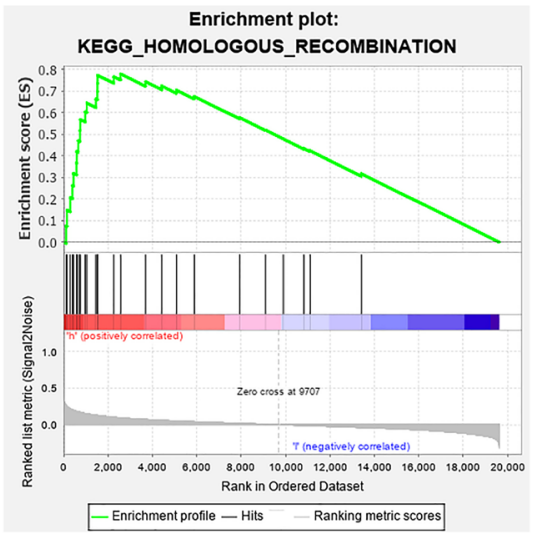

G

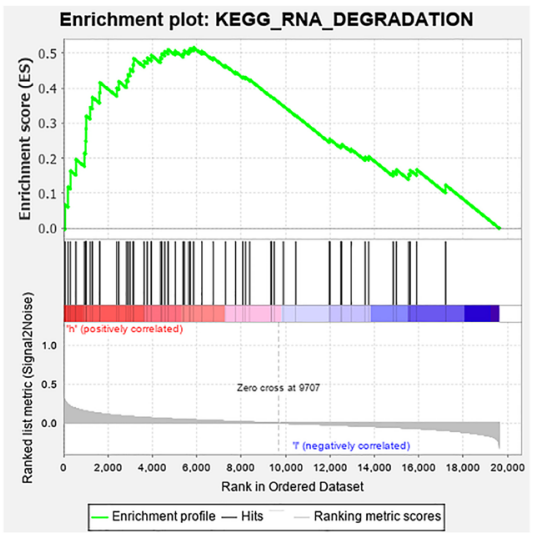

B



E

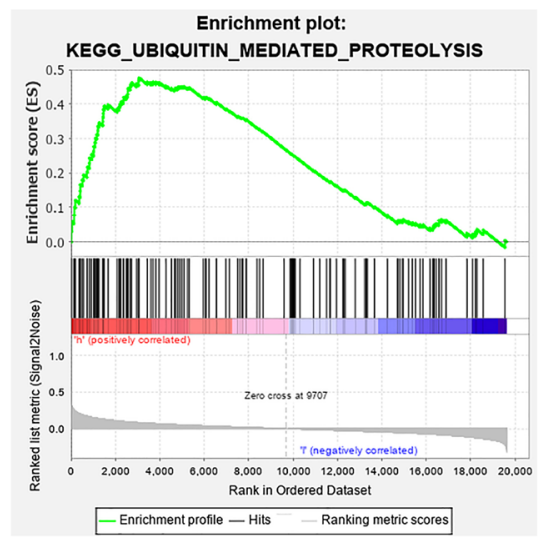

$\mathrm{H}$

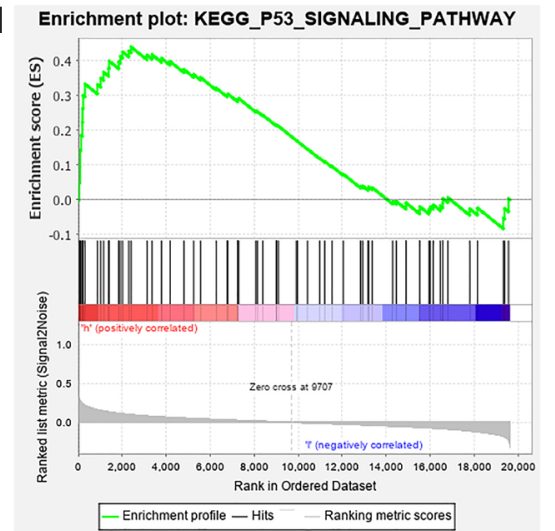

C

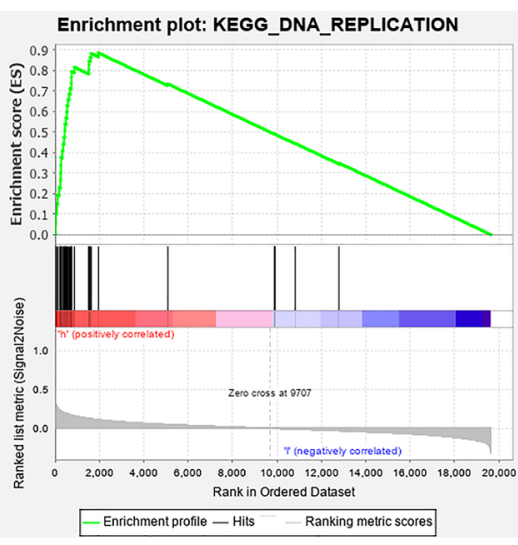

F

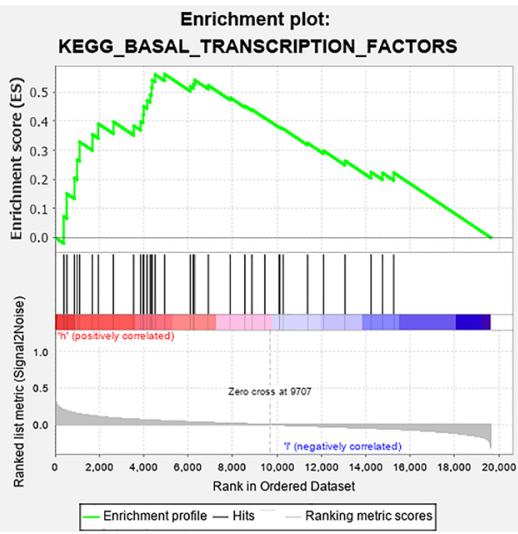

I Enrichment plot: KEGG_PATHWAYS_IN_CANCER

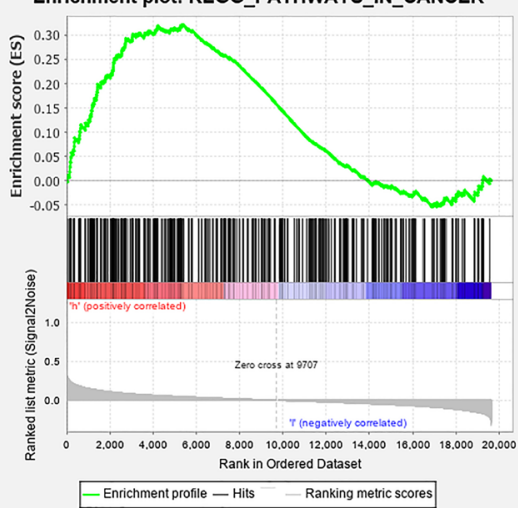

Figure 11 The signaling mechanisms involved in the risk model. (A) Cell cycle; (B) oocyte meiosis; (C) DNA replication; (D) homologous recombination; (E) ubiquitin mediated proteolysis; (F) basal transcription factors; (G) RNA degradation; (H) p53 signaling pathway; (I) pathway in cancer. 
Table 8 The signaling mechanisms involved in the high-risk group

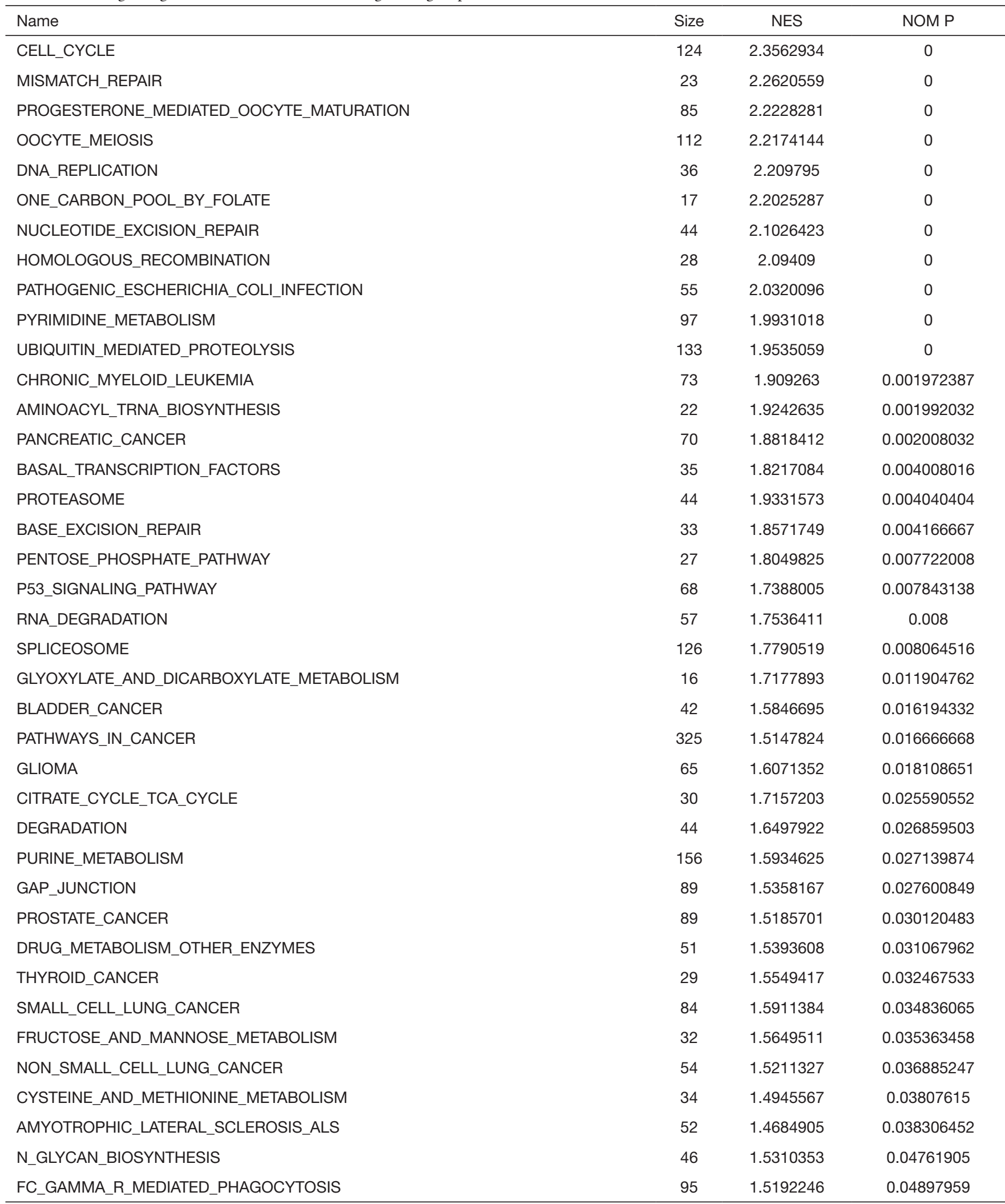

NES, Normalized Enrichment Score; NOM P, nominal P value. 
level in NCI-H1975 and SPC-A-1 cells could hinder cell proliferation, induce cell apoptosis, and inhibit tumor growth in nude mice (16). MSI1 could promote the proliferation and glucose metabolism of NSCLC cells and mediate the sensitivity to chemotherapy drugs in NSCLC cells. MSI1 could regulate protein kinase B (Akt) signaling activity (17).

Studies have shown that CCNA2 was the downstream target of miR-219-5p, and those were negatively correlated in esophageal squamous cell carcinoma (ESCC) tissues. Interference with CCNA2 expression could enhance the inhibitory effect of miR-219-5p on cell proliferation and the cell cycle (19). PLK1 inhibitor BI-6727 could induce mitotic mutations and DSB repair after radiation, thereby increasing the radiosensitivity of NSCLC cells. PLK1 silenced NSCLC cells showed that the gene expression related to DNA damage, replication, and repair were changed, including DNA-dependent protein kinase (DNAPK) and topoisomerase II $\alpha$ (TOPO2A) (18). The expression level of miR-223-5p in NSCLC tissues and cells was significantly down-regulated. The expression level of miR-223-5p was negatively correlated with the malignant degree of NSCLC. Overexpression of miR-223$5 \mathrm{p}$ could inhibit the proliferation, migration, and invasion of NSCLC cells in vitro and in vivo. This was related to the targeted regulation of E2F8 expression by miR-223-5p (19). Our study used Univariate Cox regression analysis to show that CCNA2, CHEK1, DLGAP5, E2F8, FOXM1, HIST1H2BH, HJURP, MKI67, PLK1, TPX2, and TYMS were the prognostic factors for NSCLC patients. Multivariate Cox regression analysis and AIC screening showed that HIST1H2BH and PLK1 were independent factors influencing the prognosis of NSCLC patients. It has been confirmed that some influencing factors played an important role in suppressing or promoting cancer in the progression of NSCLC (19-21), but there was still a further step to subdivide the influencing factors that need our follow-up confirmation. In addition, the risk model factors HIST1H2BH and PLK1 were overexpressed in LUAD and LUSC tissues and were related to the poor prognosis of patients. Survival analysis showed that highrisk NSCLC patients had a poor prognosis. Cox regression analysis showed that the clinical stage, $\mathrm{T}$ stage, and risk score were independent factors affecting the poor prognosis of NSCLC patients. The nomogram showed that as the risk score increases, the prognosis of NSCLC patients becomes worse. These results suggest that our risk model and nomogram have some value in predicting the prognosis of NSCLC patients.

PD-1 has been used to treat advanced cancer patients in recent years and had certain therapeutic effects (1-3). In normal lung and cancer tissues, we found that there was a correlation between the factors of the constructed risk model and the expression level of PD-1. In lung tissue, the expression level of PLK1 was significantly correlated with the expression level of PD-1. The expression level of PLK1 in normal tissues derived from LUAD and LUSC was significantly correlated with the expression level of PD-1. In LUAD and LUSC tissues, the expression level of PLK1 was significantly correlated with the expression level of PD-1. In lung tissue, the expression level of HIST1H2BH was significantly correlated with the expression level of PD-1. The expression level of HIST1H2BH in normal tissues derived from LUSC was significantly correlated with the expression level of PD-1. In LUSC tissues, the expression level of HIST1H2BH was significantly correlated with the expression level of PD-1. It further showed that HIST1H2BH and PLK1 played an important role in the progression of NSCLC.

This study analyzed the gene expression levels in the blood of PD-1 inhibitor in the treatment of NSCLC, and verified it in the TCGA database to construct the risk model and nomogram for assessing the prognosis of NSCLC. PD-1 inhibitor expression could become a potential biomarker for cancer treatment, emplying the risk model and nomogram as a guide to the patient's prognosis. The risk model factors we constructed have been verified by the multiple databases used in this study to have important clinical value, and multiple literature reported that PLK1 played an important role in the progression of NSCLC (22-24). However, this still needs further verification and will be part of our ongoing work. In general, PD-1 target molecules HIST1H2BH and PLK1 were overexpressed in LUAD and LUSC tissues. The increased expression levels of HIST1H2BH and PLK1 were related to the OS and PFS of NSCLC patients and were independent factors influencing the prognosis of NSCLC patients. In the risk model, high-risk NSCLC patients had a poor prognosis and were an independent factor influencing the poor prognosis of NSCLC patients. The high-risk group was enriched in signaling mechanisms such as cell cycle, DNA replication, homologous recombination, etc. The risk model based on PD-1 target molecules was helpful to assess the prognosis of NSCLC patients, and independent factors such as HIST1H2BH and PLK1 might become important prognostic biomarkers of NSCLC patients. 


\section{Acknowledgments}

Funding: Health Commission of Hubei Province Scientific Research Project (WJ2019Q014) and Science and Technology Development Foundation of Xiaoping Chen (CXPJJH12000002-20200).

\section{Footnote}

Reporting Checklist: The authors have completed the REMARK reporting checklist. Available at https://dx.doi. org/10.21037/atm-21-2963

Conflicts of Interest: All authors have completed the ICMJE uniform disclosure form (available at https://dx.doi. org/10.21037/atm-21-2963). The authors have no conflicts of interest to declare.

Ethical Statement: The authors are accountable for all aspects of the work in ensuring that questions related to the accuracy or integrity of any part of the work are appropriately investigated and resolved. The study was conducted in accordance with the Declaration of Helsinki (as revised in 2013).

Open Access Statement: This is an Open Access article distributed in accordance with the Creative Commons Attribution-NonCommercial-NoDerivs 4.0 International License (CC BY-NC-ND 4.0), which permits the noncommercial replication and distribution of the article with the strict proviso that no changes or edits are made and the original work is properly cited (including links to both the formal publication through the relevant DOI and the license). See: https://creativecommons.org/licenses/by-nc-nd/4.0/.

\section{References}

1. Pu N, Gao S, Yin $\mathrm{H}$, et al. Cell-intrinsic PD-1 promotes proliferation in pancreatic cancer by targeting CYR61/ CTGF via the hippo pathway. Cancer Lett 2019;460:42-53.

2. Wang B, Qin L, Ren M, et al. Effects of Combination of Anti-CTLA-4 and Anti-PD-1 on Gastric Cancer Cells Proliferation, Apoptosis and Metastasis. Cell Physiol Biochem 2018;49:260-70.

3. Andrieu GP, Shafran JS, Smith CL, et al. BET protein targeting suppresses the PD-1/PD-L1 pathway in triplenegative breast cancer and elicits anti-tumor immune response. Cancer Lett 2019;465:45-58.
4. Xia L, Huang $\mathrm{H}$, Xiao H, et al. Utilization of combined PD-L1 expression and neutrophil-to-lymphocyte ratio prior to surgery as a prognostic factor in non-small cell lung cancer with brain metastasis. Transl Cancer Res 2019;8:2864-77.

5. Wang J, Zhang R, Lin Z, et al. CDK7 inhibitor THZ1 enhances antiPD-1 therapy efficacy via the $\mathrm{p} 38 \alpha / \mathrm{MYC} /$ PD-L1 signaling in non-small cell lung cancer. J Hematol Oncol 2020;13:99.

6. Ascierto PA, Del Vecchio M, Mandalá M, et al. Adjuvant nivolumab versus ipilimumab in resected stage IIIB-C and stage IV melanoma (CheckMate 238): 4-year results from a multicentre, double-blind, randomised, controlled, phase 3 trial. Lancet Oncol 2020;21:1465-77.

7. Czarnecka AM, Rutkowski P. An update on the safety of nivolumab for the treatment of advanced melanoma. Expert Opin Drug Saf 2020;19:409-21.

8. Fujimoto D, Yomota $M$, Sekine A, et al. Nivolumab for advanced non-small cell lung cancer patients with mild idiopathic interstitial pneumonia: A multicenter, open-label single-arm phase II trial. Lung Cancer 2019;134:274-8.

9. Morita R, Okishio K, Shimizu J, et al. Real-world effectiveness and safety of nivolumab in patients with non-small cell lung cancer: A multicenter retrospective observational study in Japan. Lung Cancer 2020;140:8-18.

10. Sugawara S, Lee JS, Kang JH, et al. Nivolumab with carboplatin, paclitaxel, and bevacizumab for first-line treatment of advanced non-squamous non-small cell lung cancer. Ann Oncol 2021; undefined; undefined.

11. Hoffner B, Leighl NB, Davies M. Toxicity management with combination chemotherapy and programmed death $1 /$ programmed death ligand 1 inhibitor therapy in advanced lung cancer. Cancer Treat Rev 2020; undefined; 101979.

12. Hatae R, Chamoto K, Kim YH, et al. Combination of host immune metabolic biomarkers for the PD-1 blockade cancer immunotherapy. JCI Insight 2020;5:e133501.

13. Guo Q, Ke XX, Fang SX, et al. PAQR3 Inhibits Nonsmall Cell Lung Cancer Growth by Regulating the NFผB/p53/Bax Axis. Front Cell Dev Biol 2020; 8: 581919.

14. Siegel RL, Miller KD, Jemal A. Cancer statistics, 2020. CA Cancer J Clin 2020;70:7-30.

15. Schouten RD, Egberink L, Muller M, et al. Nivolumab in pre-treated advanced non-small cell lung cancer: long term follow up data from the Dutch expanded access program and routine clinical care. Transl Lung Cancer Res 2020;9:1736-48.

16. Xu L, Wu Q, Zhou X, et al. TRIM13 inhibited cell 
proliferation and induced cell apoptosis by regulating NF$\kappa \mathrm{B}$ pathway in non-small-cell lung carcinoma cells. Gene 2019;715:144015.

17. Lang Y, Kong X, He C, et al. Musashi1 Promotes Non-Small Cell Lung Carcinoma Malignancy and Chemoresistance via Activating the Akt Signaling Pathway. Cell Physiol Biochem 2017;44:455-66.

18. Ni Z, Yao C, Zhu X, et al. Ailanthone inhibits nonsmall cell lung cancer cell growth through repressing DNA replication via downregulating RPA1. Br J Cancer 2017;117:1621-30.

19. Ma Q. MiR-219-5p suppresses cell proliferation and cell cycle progression in esophageal squamous cell carcinoma by targeting CCNA2. Cell Mol Biol Lett 2019;24:4.

20. Yao D, Gu P, Wang Y, et al. Inhibiting polo-like kinase 1 enhances radiosensitization via modulating DNA repair proteins in non-small-cell lung cancer. Biochem Cell Biol

Cite this article as: Zhang YQ, Yuan Y, Zhang J, Lin CY, Guo JL, Liu HS, Guo Q. Evaluation of the roles and regulatory mechanisms of PD-1 target molecules in NSCLC progression. Ann Transl Med 2021;9(14):1168. doi: 10.21037/atm-21-2963
2018;96:317-25.

21. Dou L, Han K, Xiao M, et al. miR-223-5p Suppresses Tumor Growth and Metastasis in Non-Small Cell Lung Cancer by Targeting E2F8. Oncol Res 2019;27:261-8.

22. Yan L, Zhang Y, Li K, et al. miR-593-5p inhibit cell proliferation by targeting PLK1 in non small cell lung cancer cells. Pathol Res Pract 2020;216:152786.

23. Shin SB, Jang HR, Xu R, et al. Active PLK1-driven metastasis is amplified by TGF- $\beta$ signaling that forms a positive feedback loop in non-small cell lung cancer. Oncogene 2020;39:767-85.

24. Reda M, Ngamcherdtrakul W, Gu S, et al. PLK1 and EGFR targeted nanoparticle as a radiation sensitizer for non-small cell lung cancer. Cancer Lett 2019;467:9-18.

(English Language Editors: M. Bucci and J. Chapnick) 


\section{Supplementary}

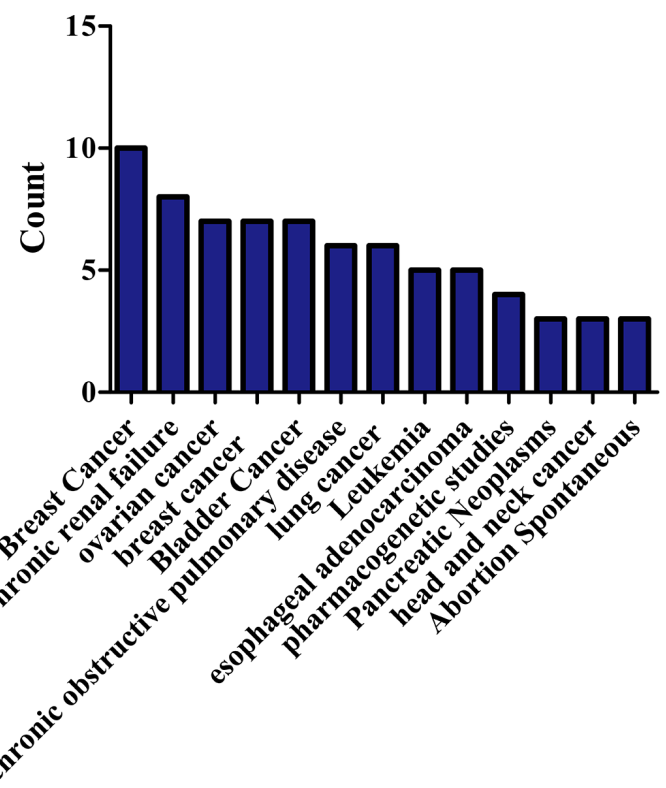

Figure S1 Disease-related to PD-1 target molecules. PD-1, programmed cell death protein 1.

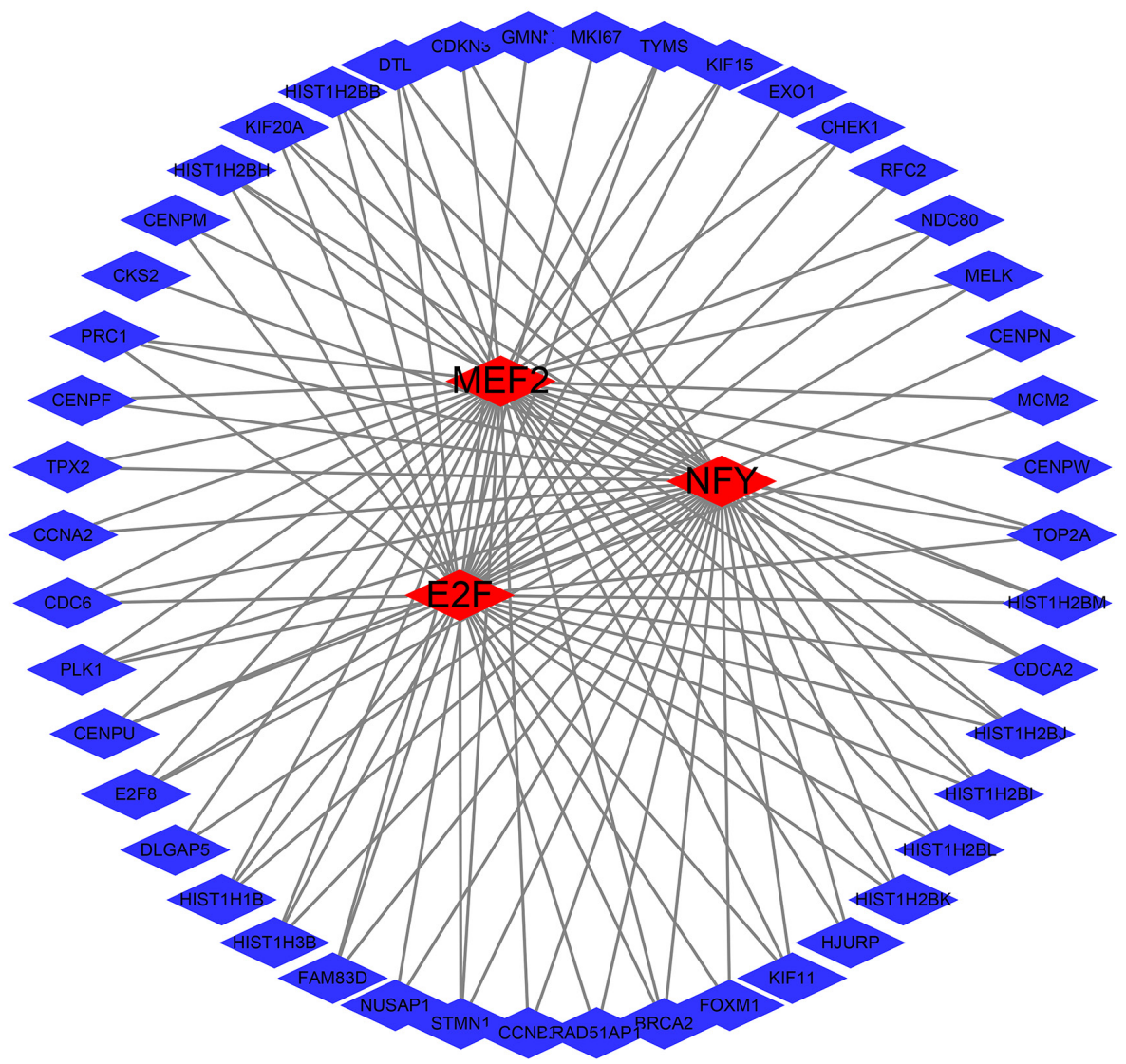

Figure S2 The relationship between transcription factors and PD-1 target molecules. PD-1, programmed cell death protein 1. 

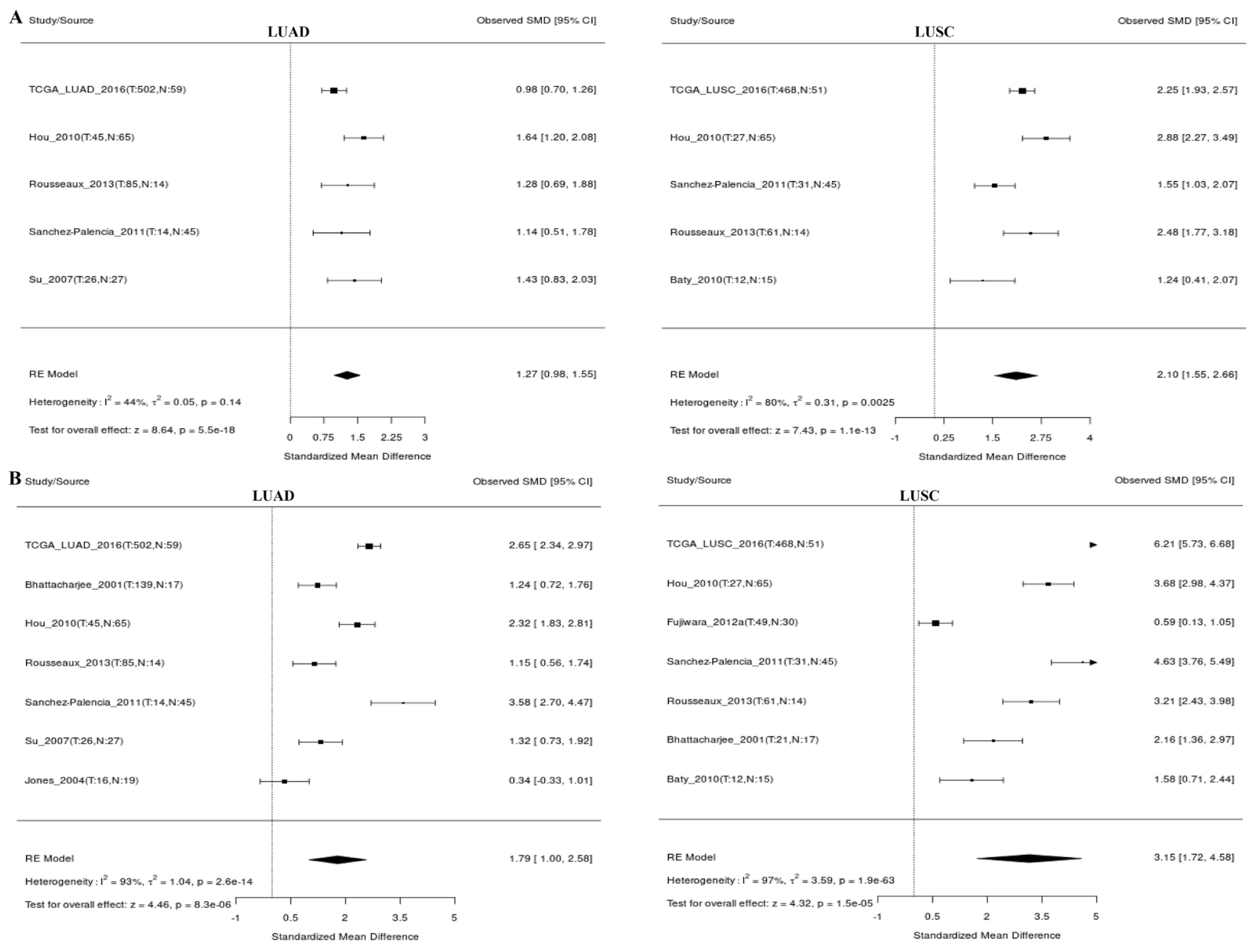

Figure S3 HIST1H2B and PLK1 were unusual expression in LUAD and LUSC via the meta-analysis. (A) HIST1H2B; (B) PLK1. LUAD, lung adenocarcinoma; LUSC, lung squamous cell carcinoma. 

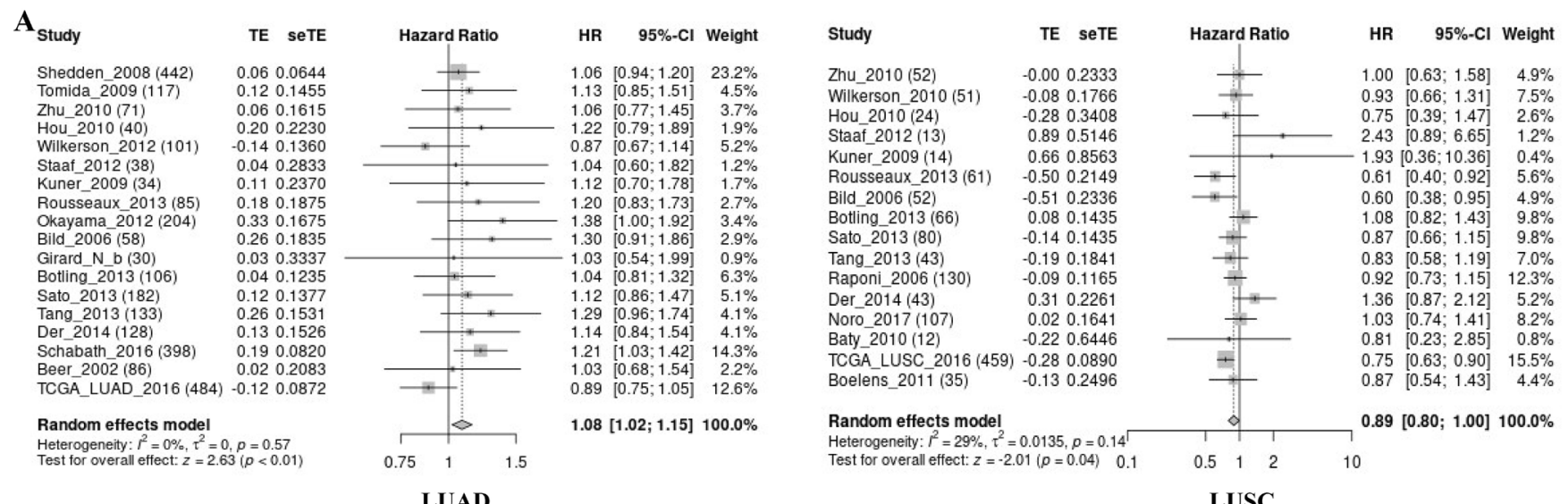

B $_{\text {study }}$

TE SeTE

LUAD

HR $95 \%$-Cl Weight

$1.21[1.07 ; 1.36] 14.2 \%$

$1.22[0.93 ; 1.61] \quad 4.9 \%$

$\begin{array}{ll}1.44[0.98 ; 2.13] & 2.7 \% \\ 0.99[0.58 ; 1.70] & 1.5 \%\end{array}$

$1.05[0.83 ; 1.34] \quad 6.2 \%$

$0.82[0.59 ; 1.15] \quad 3.6 \%$

$1.57[0.89 ; 2.77]$
$1.41[1.00 ; 2.00]$
$3.4 \%$

$\begin{array}{lll}1.41 & {[1.00 ; 2.00]} & 3.4 \% \\ 1.42 & {[1.14 ; 1.76]} & 7.0 \%\end{array}$

$\begin{array}{ll}1.29[0.87 ; 1.91] & 2.7 \% \\ 1.29[0.80 ; 2.07] & 1.9 \%\end{array}$

$\begin{array}{ll}1.29[0.80 ; 2.07] & 1.9 \% \\ 1.25[0.98 ; 1.59] & 6.0 \%\end{array}$

$\begin{array}{lll}0.26 & {[0.03 ; 2.03]} & 0.1 \% \\ 1.21 & {[0.97 ; 1.51]} & 7.0 \%\end{array}$

$1.20[0.91 ; 1.57] \quad 5.1 \%$

$1.27[0.96 ; 1.69] \quad 4.7 \%$

$1.34[1.13 ; 1.59] \quad 9.6 \%$

$\begin{array}{llll}0.91 & {[0.58 ; 1.45]} & 2.0 \% \\ 0.85 & {[0.63: 1.17]} & 4.1 \%\end{array}$

$1.39[1.15 \div 1.68] \quad 8.5 \%$

$0.94[0.66 ; 1.34] \quad 3.3 \%$

Bhattacharjee_2001 (125) -0.160 .1581$

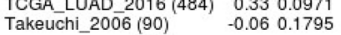

Random effects model

Heterogeneity: $r^{2}=21 \%, \tau^{2}=0.0049, p=0.1$
Test for overall effect: $z=5.40(p<0.01)$

$1.21[1.13 ; 1.29] 100.0 \%$

Figure S4 HIST1H2B and PLK1 expression level were related to the OS in LUAD and LUSC tissues via the metaanalysis. (A) HIST1H2B;

(B) PLK1. OS, overall survival; LUAD, lung adenocarcinoma; LUSC, lung squamous cell carcinoma.

$\mathbf{A}_{\text {HIST1H2BH }} \quad{ }_{2.4 \%}$

PLK1 $1.7 \%$

Genetic Alteration

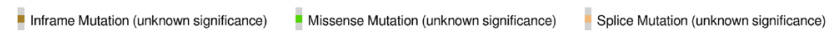

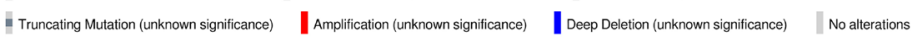

B

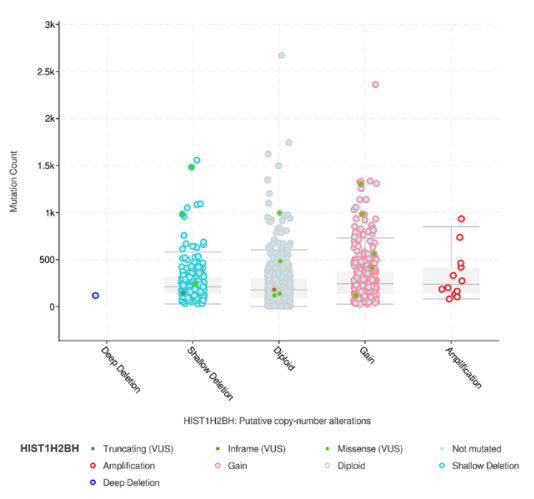

C

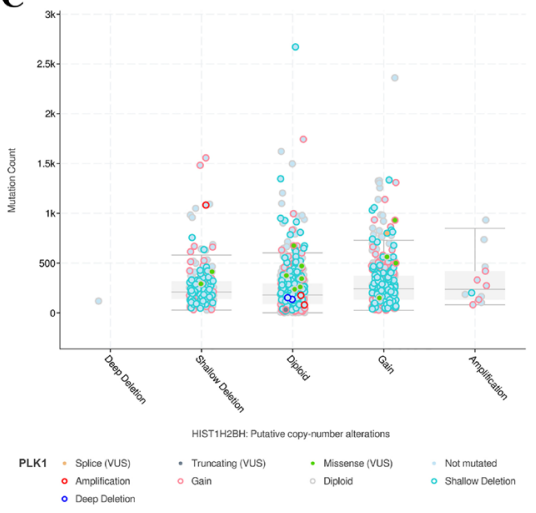

D

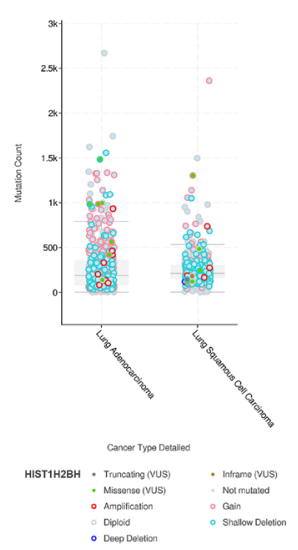

$\mathbf{E}$

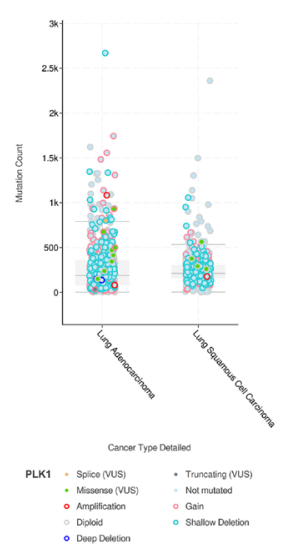

Figure S5 HIST1H2BH and PLK1 had significant mutations in NSCLC. NSCLC, non-small cell lung cancer. 

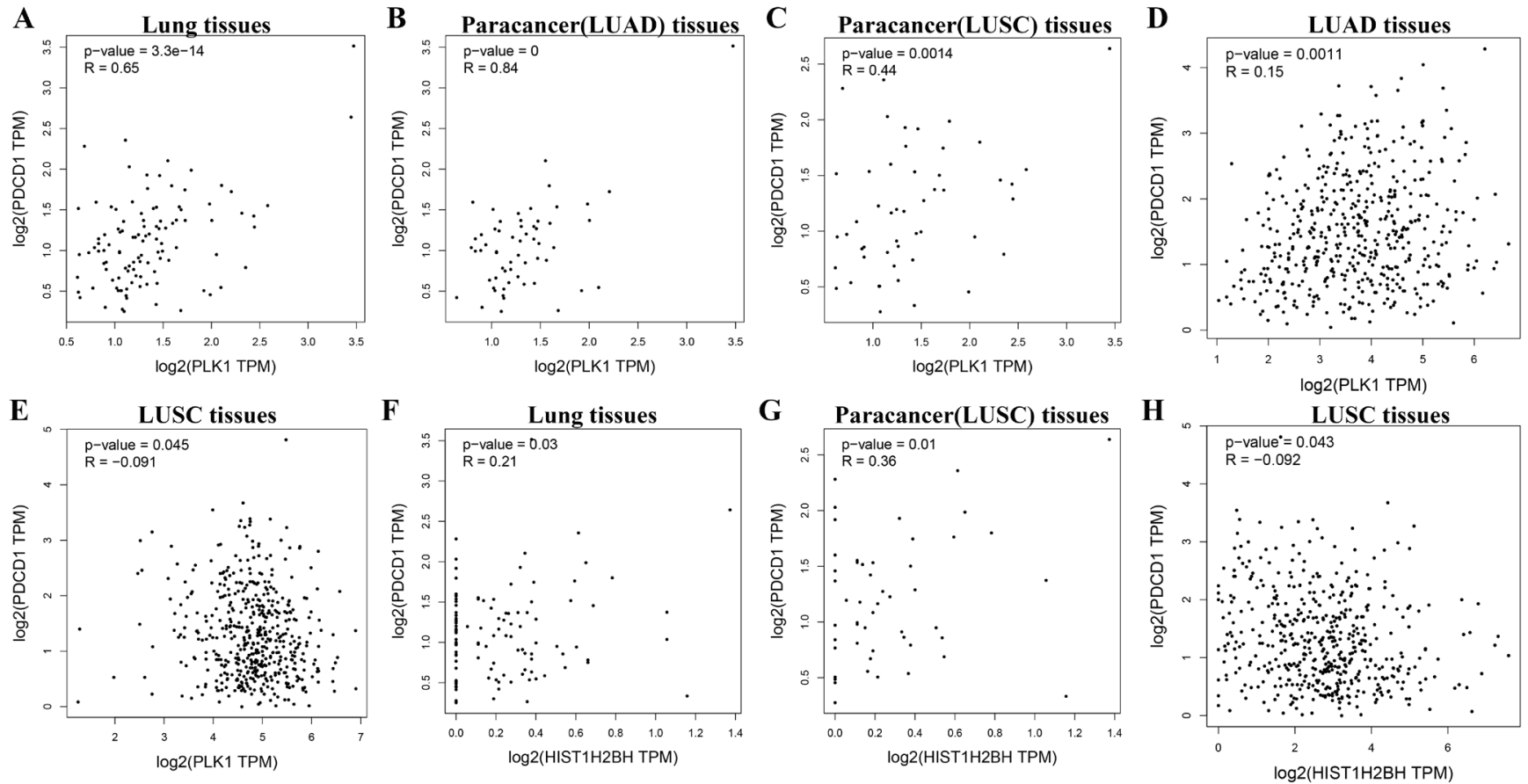

Figure S6 The expression levels of HIST1H2BH and PLK1 were correlated with PD-1 expression level. PD-1, programmed cell death protein 1. 
Table S1 The biological functions of PD-1 target molecules via the GO

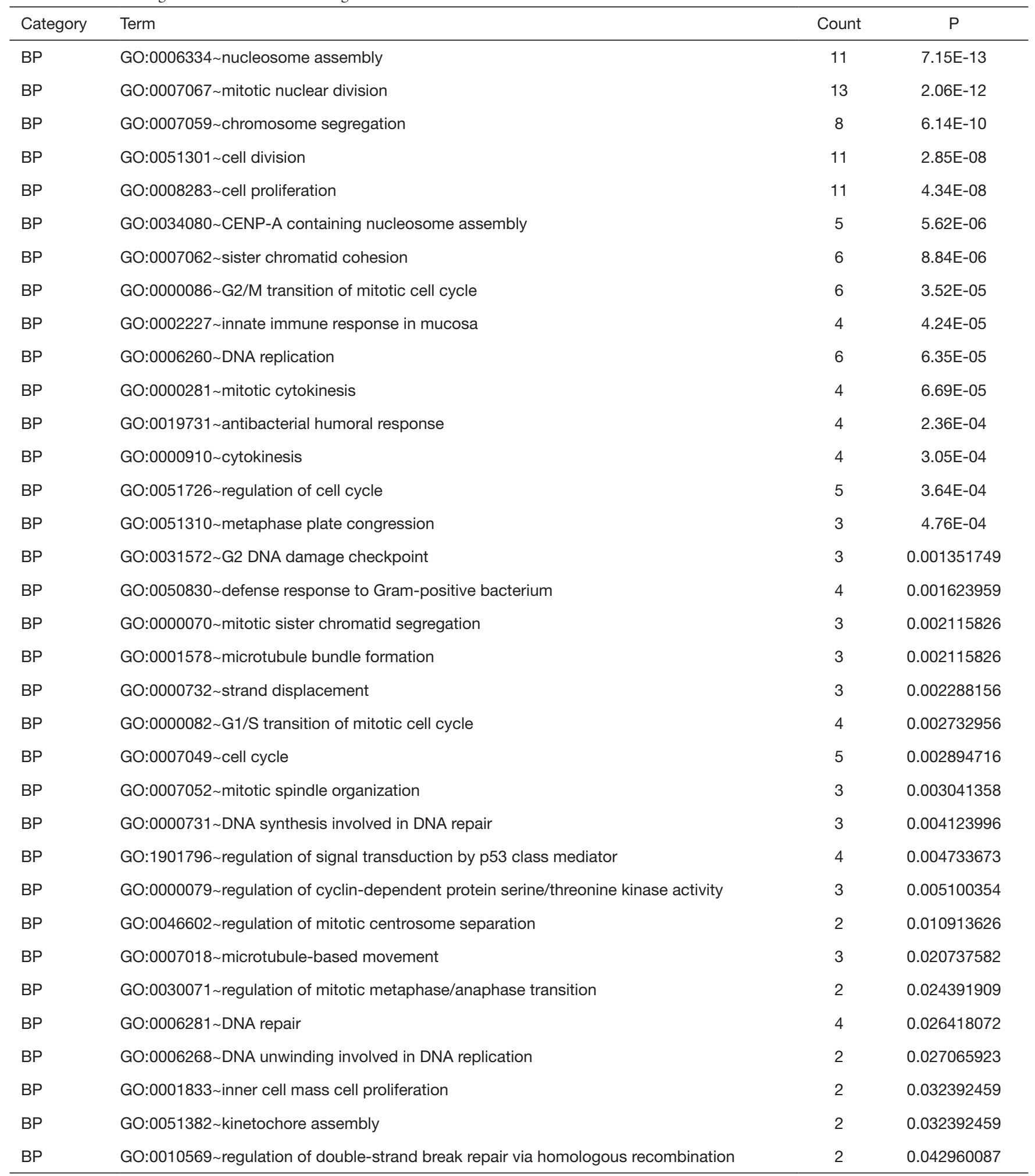

Table S1 (continued) 
Table S1 (continued)

\begin{tabular}{|c|c|c|c|}
\hline Category & Term & Count & $\mathrm{P}$ \\
\hline $\mathrm{CC}$ & GO:0000788 nuclear nucleosome & 9 & $1.44 \mathrm{E}-13$ \\
\hline CC & GO:0005819 spindle & 8 & 2.06E-08 \\
\hline CC & GO:0000775 chromosome, centromeric region & 6 & 3.11E-07 \\
\hline $\mathrm{CC}$ & GO:0005737 cytoplasm & 30 & $1.27 \mathrm{E}-06$ \\
\hline $\mathrm{CC}$ & GO:0015630 microtubule cytoskeleton & 5 & 3.90E-04 \\
\hline $\mathrm{CC}$ & GO:0005813 centrosome & 7 & $6.69 \mathrm{E}-04$ \\
\hline $\mathrm{CC}$ & GO:0005874 microtubule & 6 & 0.00108309 \\
\hline $\mathrm{CC}$ & GO:0000776 kinetochore & 4 & 0.001118872 \\
\hline $\mathrm{CC}$ & GO:0000785 chromatin & 4 & 0.001468433 \\
\hline $\mathrm{CC}$ & GO:0030496 midbody & 4 & 0.004212549 \\
\hline $\mathrm{CC}$ & GO:0000784 nuclear chromosome, telomeric region & 4 & 0.004304501 \\
\hline MF & GO:0046982 protein heterodimerization activity & 11 & $2.45 \mathrm{E}-07$ \\
\hline MF & GO:0003677 DNA binding & 18 & 4.03E-07 \\
\hline MF & GO:0019901 protein kinase binding & 9 & 4.94E-06 \\
\hline MF & GO:0008017 microtubule binding & 7 & $1.56 \mathrm{E}-05$ \\
\hline MF & GO:0005515 protein binding & 36 & $1.49 \mathrm{E}-04$ \\
\hline MF & GO:0005524 ATP binding & 12 & 0.001252552 \\
\hline MF & GO:0042393 histone binding & 4 & 0.003930198 \\
\hline MF & GO:0008022 protein C-terminus binding & 4 & 0.011807477 \\
\hline MF & GO:0003777 microtubule motor activity & 3 & 0.018448317 \\
\hline MF & GO:0042826 histone deacetylase binding & 3 & 0.029009124 \\
\hline
\end{tabular}

PD-1, programmed cell death protein 1; GO, gene ontology; BP, biological processes; CC, cellular component; MF, molecular function. 